\title{
Complete six-gluon disk amplitude in superstring theory
}

\author{
Stephan Stieberger ${ }^{\mathrm{a}, \mathrm{b}, *}$, Tomasz R. Taylor ${ }^{\mathrm{a}, \mathrm{c}}$ \\ ${ }^{a}$ Max-Planck-Institut für Physik, Werner-Heisenberg-Institut, 80805 München, Germany \\ ${ }^{\mathrm{b}}$ Max-Planck-Institut für Gravitationsphysik, Albert-Einstein-Institut, 14476 Potsdam, Germany \\ c Department of Physics, Northeastern University, Boston, MA 02115, USA
}

Received 2 April 2008; received in revised form 22 April 2008; accepted 28 April 2008

Available online 7 May 2008

\begin{abstract}
We evaluate all next-to-maximal helicity violating (NMHV) six-gluon amplitudes in type I open superstring theory in four dimensions, at the disk level, to all orders in $\alpha^{\prime}$. Although the computation utilizes supersymmetric Ward identities, the result holds for all compactifications, even for those that break supersymmetry and is completely model-independent. Together with the maximally helicity violating (MHV) amplitudes presented in the previous work, our results provide the complete six-gluon disk amplitude.

(c) 2008 Elsevier B.V. All rights reserved.
\end{abstract}

\section{Introduction}

Multi-gluon scattering amplitudes are important from both theoretical and experimental points of view because they describe the processes underlying hadronic jet production at high energy colliders. Up to the energies accessible to the existing accelerators, there is an excellent agreement between experimental data and the amplitudes calculated in the framework of perturbative Quantum Chromodynamics (QCD). If in the upcoming Large Hadron Collider (LHC) experiments any discrepancy is discovered between QCD and the observed jet cross sections, it will be interpreted as a signal of new physics beyond the standard model.

Among the extensions of the standard model, superstring theory stands out as one of the boldest ones because it incorporates gravity and covers a huge span of energies, up to the Planck mass. However, the fundamental energy scale is the string mass, which need not necessarily to be as high provided that the Universe contains some large extra dimensions [1,2]. If the string scale

\footnotetext{
* Corresponding author at: Max-Planck-Institut für Physik, Werner-Heisenberg-Institut, 80805 München, Germany.

E-mail address: stieberg@mppmu.mpg.de (S. Stieberger).
} 
is within, or not too far above the range of LHC energies, the effects of Regge excitations may be observable, and a direct experimental proof of superstring theory can be at hand. In particular, the multi-gluon amplitudes will be affected by the so-called $\alpha^{\prime}$ corrections and the measurement of the corresponding jet cross sections can reveal some spectacular signals of superstring theory.

In a recent series of papers [3-6], we developed a formalism for computing $N$-gluon amplitudes at the disk level, i.e., at the leading order in the string/gauge coupling constant but to all orders in $\alpha^{\prime}$. The most important property of these leading contributions is that they are completely model-independent. These amplitudes are very robust because they hold for arbitrary compactifications of superstring theory from ten to four dimensions, including those that break supersymmetry. ${ }^{1}$ The formalism combines the use of traditional supersymmetry and helicity techniques together with some elements of the theory of multiple hypergeometric integrals that allow expressing the final results in terms of $(N-3)$ ! generalized hypergeometric functions of kinematic invariants. This is particularly effective when applied to the maximally helicity violating [9] (MHV) amplitudes [6]. Next-to-maximal helicity violating (NMHV) amplitudes which appear starting from $N=6$ seem to have a more complicated structure. In this work, we study the case of six-gluon NMHV amplitudes. We obtain some relatively simple expressions for NMHV amplitudes which complement the MHV amplitudes presented in [4-6], providing the full six-gluon disk amplitude.

The paper is organized as follows. In Section 2, we present supersymmetry (SUSY) relations that allow expressing all three independent six-gluon NMHV amplitudes in terms of the amplitudes involving four scalars and two gluons, four scalars and two gauginos, and six scalars. In Section 3, we evaluate these auxiliary amplitudes and express them in terms of certain hypergeometric integrals. In Section 4, we combine them according to SUSY relations and obtain explicit expressions for all NMHV amplitudes. We show that the leading order of the expansion in powers of $\alpha^{\prime}$ correctly reproduces the QCD result. In Appendices A, B, we list the relevant hypergeometric functions and give their $\alpha^{\prime}$-expansions up to the next-to-leading order $\mathcal{O}\left(\alpha^{\prime 2}\right)$ with respect to the leading (QCD) contributions.

\section{SUSY relations for NMHV amplitudes}

The full six-gluon NMHV amplitude can be constructed from three partial subamplitudes $[10,11]$, each associated to the same Chan-Paton factor $\operatorname{Tr}\left(T^{a_{1}} \cdots T^{a_{6}}\right)$, but characterized by three inequivalent helicity orderings:

$$
\begin{aligned}
& A^{Y} \equiv A\left(g_{1}^{-}, g_{2}^{-}, g_{3}^{+}, g_{4}^{+}, g_{5}^{-}, g_{6}^{+}\right), \\
& A^{X} \equiv A\left(g_{1}^{+}, g_{2}^{+}, g_{3}^{-}, g_{4}^{-}, g_{5}^{-}, g_{6}^{+}\right), \\
& A^{Z} \equiv A\left(g_{1}^{-}, g_{2}^{+}, g_{3}^{-}, g_{4}^{+}, g_{5}^{-}, g_{6}^{+}\right) .
\end{aligned}
$$

These amplitudes will be expressed in terms of the following amplitudes with the gluons replaced by scalars or fermions:

$$
\begin{array}{ll}
A_{\lambda}^{Y} \equiv A\left(\phi_{1}^{-}, \phi_{2}^{-}, \phi_{3}^{+}, \phi_{4}^{+}, \lambda_{5}^{-}, \lambda_{6}^{+}\right), & A_{s}^{Y} \equiv A\left(\phi_{1}^{-}, \phi_{2}^{-}, \phi_{3}^{+}, \phi_{4}^{+}, \phi_{5}^{-}, \phi_{6}^{+}\right), \\
A_{\lambda}^{X} \equiv A\left(\phi_{1}^{+}, \phi_{2}^{+}, \phi_{3}^{-}, \phi_{4}^{-}, \lambda_{5}^{-}, \lambda_{6}^{+}\right), & A_{s}^{X} \equiv A\left(\phi_{1}^{+}, \phi_{2}^{+}, \phi_{3}^{-}, \phi_{4}^{-}, \phi_{5}^{-}, \phi_{6}^{+}\right),
\end{array}
$$

\footnotetext{
1 The difference between supersymmetric and non-supersymmetric amplitudes appears at the one-loop level. In particular, the presence of non-supersymmetric dimension six operator $\operatorname{Tr}\left(F_{\mu \nu} F^{v \rho} F_{\rho \mu}\right)$, where $F$ is the gauge field strength tensor, may have some interesting phenomenological consequences [7,8].
} 


$$
A_{\lambda}^{Z} \equiv A\left(\phi_{1}^{-}, \phi_{2}^{+}, \phi_{3}^{-}, \phi_{4}^{+}, \lambda_{5}^{-}, \lambda_{6}^{+}\right), \quad A_{s}^{Z} \equiv A\left(\phi_{1}^{-}, \phi_{2}^{+}, \phi_{3}^{-}, \phi_{4}^{+}, \phi_{5}^{-}, \phi_{6}^{+}\right),
$$

and the amplitudes with four gluons replaced by scalars:

$$
\begin{aligned}
& A_{g}^{Y} \equiv A\left(\phi_{1}^{-}, \phi_{2}^{-}, \phi_{3}^{+}, \phi_{4}^{+}, g_{5}^{-}, g_{6}^{+}\right), \\
& A_{g}^{X} \equiv A\left(\phi_{1}^{+}, \phi_{2}^{+}, \phi_{3}^{-}, \phi_{4}^{-}, g_{5}^{-}, g_{6}^{+}\right), \\
& A_{g}^{Z} \equiv A\left(\phi_{1}^{-}, \phi_{2}^{+}, \phi_{3}^{-}, \phi_{4}^{+}, g_{5}^{-}, g_{6}^{+}\right)
\end{aligned}
$$

Here, $\phi$ is the scalar component of $\mathcal{N}=2$ gauge supermultiplet and $\lambda$ is one of the two gauginos [6].

Recently, we showed [6] that all field-theoretical SUSY relations [12,13] between scattering amplitudes hold also in superstring theory at the disk level, to all orders in $\alpha^{\prime}$. Actually, we find most useful the relations already used in the original computation of the six-gluon QCD amplitudes [14]. In order to write these relations down, we first introduce the following kinematic variables

$$
\begin{array}{lll}
Y=k_{3}+k_{4}+k_{6}, & \alpha_{Y}=-\langle 12\rangle[34][6|Y| 5\rangle, & y=\langle 12\rangle[34] Y^{2}, \\
X=k_{1}+k_{2}+k_{6}, & \alpha_{X}=-[12]\langle 34\rangle[6|X| 5\rangle, & x=[12]\langle 34\rangle X^{2}, \\
Z=k_{2}+k_{4}+k_{6}, & \alpha_{Z}=-\langle 13\rangle[24][6|Z| 5\rangle, & z=\langle 13\rangle[24] Z^{2},
\end{array}
$$

depending on the momenta $k_{1}, k_{2}, \ldots, k_{6}$. Here, we used the standard notation $[10,11]$ for spinor products, in particular:

$$
[6|Y| 5\rangle=[63]\langle 35\rangle+[64]\langle 45\rangle, \quad \text { etc. }
$$

For the scalar products of momenta, we use the notation of [5]:

$$
\begin{aligned}
& s_{i j}=2 \alpha^{\prime} k_{i} k_{j}, \quad s_{i}=\alpha^{\prime}\left(k_{i}+k_{i+1}\right)^{2}, \\
& t_{i}=\alpha^{\prime}\left(k_{i}+k_{i+1}+k_{i+2}\right)^{2} \quad(i+6 \equiv i) .
\end{aligned}
$$

All scalar products $s_{i j}$ can be expressed in terms of $s_{1}, s_{2}, s_{3}, s_{4}, s_{5}, s_{6}$ and $t_{1}, t_{2}, t_{3}$ [5], e.g., $s_{12}=s_{1}$, etc. Now the SUSY relations can be written as

$$
\begin{aligned}
& A^{Y}=\frac{\alpha^{\prime 4}}{s_{12}^{2} s_{34}^{2}}\left(y^{2} A_{g}^{Y}-2 y \alpha_{Y} A_{\lambda}^{Y}+\alpha_{Y}^{2} A_{s}^{Y}\right), \\
& A^{X}=\frac{\alpha^{\prime 4}}{s_{12}^{2} s_{34}^{2}}\left(x^{2} A_{g}^{X}-2 x \alpha_{X} A_{\lambda}^{X}+\alpha_{X}^{2} A_{s}^{X}\right), \\
& A^{Z}=\frac{\alpha^{\prime 4}}{s_{13}^{2} s_{24}^{2}}\left(z^{2} A_{g}^{Z}-2 z \alpha_{Z} A_{\lambda}^{Z}+\alpha_{Z}^{2} A_{s}^{Z}\right),
\end{aligned}
$$

where the factors $\alpha^{\prime 4}$ appear artificially, due to the choice of string units in (2.6). Note that the latter two relations $(X, Z)$ follow from the first one $(Y)$ by the replacements $(1 \leftrightarrow 4,2 \leftrightarrow 3)$ and $(2 \leftrightarrow 3)$, respectively. In Section 3, we evaluate the amplitudes appearing on the r.h.s. of Eq. (2.7). 


\section{Six-point disk scattering of scalars, gauginos and vectors}

In this section, we compute the six-point string amplitudes (2.2) and (2.3) involving scalars, gauginos and vectors of the four-dimensional $\mathcal{N}=2$ vector multiplet. The world-sheet of the string $S$-matrix is described by a disk with all external states $\Phi^{a}$ created by vertex operators $V_{\Phi^{a}}$ at the boundary the disk. In the notation of Refs. [4-6], the partial amplitude associated to the $\operatorname{Tr}\left(T^{a_{1}} \cdots T^{a_{6}}\right)$ Chan-Paton factor takes the form:

$$
\begin{aligned}
A & \left(\Phi^{a_{1}}, \Phi^{a_{2}}, \Phi^{a_{3}}, \Phi^{a_{4}}, \Phi^{a_{4}}, \Phi^{a_{6}}\right) \\
& =V_{\mathrm{CKG}}^{-1} \int_{z_{1}<\cdots<z_{6}}\left(\prod_{k=1}^{6} d z_{k}\right)\left\langle V_{\Phi^{a_{1}}}\left(z_{1}\right) V_{\Phi^{a_{2}}}\left(z_{2}\right) V_{\Phi^{a_{3}}}\left(z_{3}\right) V_{\Phi^{a_{4}}}\left(z_{4}\right) V_{\Phi^{a_{5}}}\left(z_{5}\right) V_{\Phi^{a_{6}}}\left(z_{6}\right)\right\rangle .
\end{aligned}
$$

In order to cancel the total background ghost charge in the disk correlator (3.1), the vertex operators have to be chosen in the appropriate ghost picture. Furthermore, in Eq. (3.1), the factor $V_{\mathrm{CKG}}$ accounts for the volume of the conformal Killing group of the disk after choosing the conformal gauge. It will be canceled by fixing three vertex positions and introducing the respective $c$-ghost correlator. The gaugino vertex operators, in the $(-1 / 2)$-ghost picture, are $^{2}$ :

$$
\begin{aligned}
& V_{\lambda^{a, I}}^{(-1 / 2)}(z, u, k)=g_{\lambda} T^{a} e^{-\phi / 2} u^{\alpha} S_{\alpha} \Sigma^{I} e^{i k_{\rho} X^{\rho}}, \\
& V_{\bar{\lambda}^{a, I}}^{(-1 / 2)}(z, u, k)=g_{\lambda} T^{a} e^{-\phi / 2} \bar{u}_{\dot{\beta}} S^{\dot{\beta}} \bar{\Sigma}^{I} e^{i k_{\rho} X^{\rho}}, \quad I=1,2 .
\end{aligned}
$$

Here $S_{\alpha}, S_{\dot{\alpha}}$ are the spin fields with the indices $\alpha$ (or $\dot{\alpha}$ ) denoting negative (positive) chirality in four dimensions. Furthermore, $\phi$ is the scalar bosonizing the superghost system. In the above definitions, $T^{a}$ are the Chan-Paton factors accounting for the gauge degrees of freedom of the two open string ends. The on-shell constraints $k^{2}=0, \not k u=0$ are imposed.

For $\mathcal{N}=2$ space-time SUSY the internal SCFT splits into two pieces. One piece is the $c=3$ superconformal algebra, which corresponds to a torus compactificaton with the two complex internal fermions $\Psi^{\mp}=e^{ \pm i H_{3}}$. The second piece represents a $c=6$ superconformal algebra, which contains the $S U(2)$ currents $J_{3}=i \partial H, J^{12}=e^{i \sqrt{2} H}$ and $J^{21}=e^{-i \sqrt{2} H}$ [16]. The (internal) Ramond fields $\Sigma^{I}$ may be expressed by these bosonic fields $H_{3}$ and $H$ :

$$
\Sigma^{1}=e^{\frac{i}{2} H_{3}} e^{\frac{i}{\sqrt{2}} H}, \quad \Sigma^{2}=e^{\frac{i}{2} H_{3}} e^{-\frac{i}{\sqrt{2}} H} .
$$

Finally, the vertex operators $V_{\phi^{a, \pm}}(z, k)$ for the scalars and for the vectors $V_{A^{a}}(z, \xi, k)$ can be found in Section 3 of [6].

The six-point correlator in the integrand of (3.1) is evaluated by performing all possible Wick contractions. All three and four-point fermionic correlators involving fermions and spin fields are given in $[6,17,18]$, while an important five-point correlator will be computed below. Because of the $\operatorname{PSL}(2, \mathbf{R})$ invariance on the disk, we can fix three positions of the vertex operators. A convenient choice respecting the ordering $z_{1}<\cdots<z_{6}$ is

$$
z_{1}=-z_{\infty}=-\infty, \quad z_{2}=0, \quad z_{3}=1,
$$

\footnotetext{
2 The open string vertex couplings are $g_{\phi}=\left(2 \alpha^{\prime}\right)^{1 / 2} g_{\mathrm{YM}}, g_{\lambda}=\left(2 \alpha^{\prime}\right)^{1 / 2} \alpha^{\prime 1 / 4} g_{\mathrm{YM}}$, and $g_{A}=\left(2 \alpha^{\prime}\right)^{1 / 2} g_{\mathrm{YM}}$ for the scalar, gaugino and vector, respectively. The $D=4$ gauge coupling $g_{\mathrm{YM}}$ can be expressed in terms of the ten-dimensional gauge coupling $g_{10}$ and the dilaton field $\phi_{10}$ through the relation $g_{\mathrm{YM}}=g_{10} e^{\phi_{10} / 2}[15]$.
} 
which implies the ghost factor $\left\langle c\left(z_{1}\right) c\left(z_{2}\right) c\left(z_{3}\right)\right\rangle=-z_{\infty}^{2}$. The remaining three vertex positions take arbitrary values inside the integration domain $1<z_{4}<z_{5}<z_{6}<\infty$. The latter is parameterized by $z_{4}=x^{-1}, z_{5}=(x y)^{-1}$ and $z_{6}=(x y z)^{-1}$, with $0<x, y, z<1$. Generally for this choice, the integrand of (3.1) contains the common factor $[3,5]$

$$
\begin{aligned}
\mathcal{I}(x, y, z)= & x^{s_{2}} y^{t_{2}} z^{s_{6}}(1-x)^{s_{3}}(1-y)^{s_{4}}(1-z)^{s_{5}} \\
& \times(1-x y)^{t_{3}-s_{3}-s_{4}}(1-y z)^{t_{1}-s_{4}-s_{5}}(1-x y z)^{s_{1}+s_{4}-t_{1}-t_{3}} .
\end{aligned}
$$

The resulting integrals represent generalized Euler integrals and integrate to multiple Gaussian hypergeometric functions $[3,5]$.

In order to correctly normalize the amplitudes, some additional factors have to be taken into account. They stem from determinants and Jacobians of certain path integrals. On the disk, the net result of those contributions is an additional factor of $C_{D_{2}}=\frac{1}{2 g_{\mathrm{YM}^{\alpha^{\prime}}}^{2}}$ which must be included in all disk correlators [15].

\subsection{Four scalars and two gauginos}

First, we compute the three subamplitudes (2.2) involving four scalars and two gauginos: $A_{\lambda}^{Y}$, $A_{\lambda}^{X}$ and $A_{\lambda}^{Z}$. To that end, we evaluate the amplitude (3.1) with the following correlator:

$$
\left\langle V_{\phi^{a_{1}}}^{(0)}\left(z_{1}, k_{1}\right) V_{\phi^{a_{2}}}^{(0)}\left(z_{2}, k_{2}\right) V_{\phi^{a_{3}}}^{(0)}\left(z_{3}, k_{3}\right) V_{\phi^{a_{4}}}^{(-1)}\left(z_{4}, k_{4}\right) V_{\lambda^{a_{5}, I}}^{(-1 / 2)}\left(z_{5}, u_{5}, k_{5}\right) V_{\bar{\lambda}^{a_{6}, J}}^{(-1 / 2)}\left(z_{6}, \bar{u}_{6}, k_{6}\right)\right\rangle,
$$

for the helicity configurations $Y, X$ and $Z$.

To compute (3.6), we need ${ }^{3}$ the five-point function

$$
\begin{aligned}
& \left\langle\psi^{\lambda_{1}}\left(z_{1}\right) \psi^{\lambda_{2}}\left(z_{2}\right) \psi^{\lambda_{3}}\left(z_{3}\right) S_{\alpha}\left(z_{4}\right) S_{\dot{\beta}}\left(z_{5}\right)\right\rangle \\
& =\frac{1}{\sqrt{2}}\left(z_{14} z_{15} z_{24} z_{25} z_{34} z_{35}\right)^{-1 / 2} \\
& \quad \times\left\{\frac{z_{24} z_{35}}{z_{23}} \sigma_{\alpha \dot{\beta}}^{\lambda_{1}} \delta^{\lambda_{2} \lambda_{3}}-\frac{z_{14} z_{35}}{z_{13}} \sigma_{\alpha \dot{\beta}}^{\lambda_{2}} \delta^{\lambda_{1} \lambda_{3}}+\frac{z_{14} z_{25}}{z_{12}} \sigma_{\alpha \dot{\beta}}^{\lambda_{3}} \delta^{\lambda_{1} \lambda_{2}}+\frac{1}{2} z_{45}\left(\sigma^{\lambda_{1}} \bar{\sigma}^{\lambda_{2}} \sigma^{\lambda_{3}}\right)_{\alpha \dot{\beta}}\right\},
\end{aligned}
$$

which may be derived by studying its singular behavior and by using equations written in [6]. The last term in (3.7) may also be rewritten thanks to the identity $: i \epsilon^{\lambda_{1} \lambda_{2} \lambda_{3} \lambda_{1}} \sigma_{\lambda}=\sigma^{\lambda_{1}} \bar{\sigma}^{\lambda_{2}} \sigma^{\lambda_{3}}+$

3 Throughout this article we adapt to the notation and spinor algebra of the book of Wess and Bagger. In particular, spinor indices are raised and lowered with the anti-symmetric tensors $\epsilon_{\alpha \beta}$ and $\epsilon^{\dot{\alpha} \dot{\beta}}$. Besides spinor products are defined to be $\chi \eta=\chi^{\alpha} \epsilon_{\alpha \beta} \eta^{\beta}\left(\bar{\chi} \bar{\eta}=\bar{\chi}_{\dot{\alpha}} \epsilon^{\dot{\alpha} \dot{\beta}} \bar{\eta}_{\dot{\beta}}\right)$ for some spinors $\chi, \eta(\bar{\chi}, \bar{\eta})$.

4 With this identity the correlator (3.7) becomes:

$$
\begin{aligned}
& \left\langle\psi^{\lambda_{1}}\left(z_{1}\right) \psi^{\lambda_{2}}\left(z_{2}\right) \psi^{\lambda_{3}}\left(z_{3}\right) S_{\alpha}\left(z_{4}\right) S_{\dot{\beta}}\left(z_{5}\right)\right\rangle \\
& =\frac{1}{2 \sqrt{2}}\left(z_{14} z_{15} z_{24} z_{25} z_{34} z_{35}\right)^{-1 / 2}\left\{i \epsilon^{\lambda_{1} \lambda_{2} \lambda_{3} \lambda}\left(\sigma_{\lambda}\right)_{\alpha \dot{\beta}} z_{45}\right. \\
& \left.\quad+\frac{\sigma_{\alpha \dot{\beta}}^{\lambda_{1}} \delta^{\lambda_{2} \lambda_{3}}}{z_{23}}\left(z_{24} z_{35}+z_{25} z_{34}\right)-\frac{\sigma_{\alpha \dot{\beta}}^{\lambda_{2}} \delta^{\lambda_{1} \lambda_{3}}}{z_{13}}\left(z_{14} z_{35}+z_{15} z_{34}\right)+\frac{\sigma_{\alpha \dot{\beta}}^{\lambda_{3}} \delta^{\lambda_{1} \lambda_{2}}}{z_{12}}\left(z_{14} z_{25}+z_{15} z_{24}\right)\right\} .
\end{aligned}
$$


$\delta^{\lambda_{2} \lambda_{3}} \sigma^{\lambda_{1}}-\delta^{\lambda_{1} \lambda_{3}} \sigma^{\lambda_{2}}+\delta^{\lambda_{1} \lambda_{2}} \sigma^{\lambda_{3}}$. Furthermore, we need the two correlators of internal fields:

$$
\begin{aligned}
& \left\langle\Psi\left(z_{1}\right) \bar{\Psi}\left(z_{2}\right) \Sigma^{I}\left(z_{3}\right) \bar{\Sigma}^{J}\left(z_{4}\right)\right\rangle=\delta^{I J} z_{12}^{-1} z_{34}^{-3 / 4}\left(\frac{z_{13} z_{24}}{z_{14} z_{23}}\right)^{1 / 2}, \\
& \left\langle\Psi\left(z_{1}\right) \Psi\left(z_{2}\right) \bar{\Psi}\left(z_{3}\right) \bar{\Psi}\left(z_{4}\right) \Sigma^{I}\left(z_{5}\right) \bar{\Sigma}^{J}\left(z_{6}\right)\right\rangle=\delta^{I J} \frac{z_{12} z_{34}}{z_{13} z_{14} z_{23} z_{24}}\left(\frac{z_{15} z_{25} z_{36} z_{46}}{z_{16} z_{26} z_{35} z_{45}}\right)^{1 / 2} z_{56}^{-3 / 4} .
\end{aligned}
$$

All remaining correlators appearing in (3.6) are basic and can be found in [6].

After assembling everything in the correlators (3.6), each of the partial amplitudes $Y, X$ and $Z$ takes the form

$$
A_{\lambda}=4 \alpha^{\prime 2} g_{\mathrm{YM}}^{4}\left([6|1| 5\rangle L_{1}+[6|2| 5\rangle L_{2}+[6|3| 5\rangle L_{3}-\alpha^{\prime}[6|3| 2\rangle[2|1| 5\rangle L_{4}\right),
$$

with the set of four functions $L_{i} \in\left\{L_{i}^{Y}, L_{i}^{X}, L_{i}^{Z}\right\}, i=1,2,3,4$, specific to the three helicity configurations $Y, X$ and $Z$, respectively. The integral representations of these functions are given in Appendix A. Actually, the kinematic factor in front of $L_{4}$ can be expressed in terms of those in front of $L_{1,2,3}$, and the result (3.9) can be simplified to

$$
A_{\lambda}=4 \alpha^{\prime 2} g_{\mathrm{YM}}^{4}\left([6|1| 5\rangle H_{1}+[6|2| 5\rangle H_{2}+[6|3| 5\rangle H_{3}\right),
$$

where

$$
\begin{aligned}
& H_{1}=L_{1}-\frac{L_{4}}{2 s_{5}}\left(s_{25} s_{36}-s_{26} s_{35}+s_{23} s_{56}\right), \\
& H_{2}=L_{2}-\frac{L_{4}}{2 s_{5}}\left(s_{16} s_{35}-s_{15} s_{36}-s_{13} s_{56}\right), \\
& H_{3}=L_{3}-\frac{L_{4}}{2 s_{5}}\left(s_{15} s_{26}-s_{16} s_{25}+s_{12} s_{56}\right) .
\end{aligned}
$$

\subsection{Six scalars}

Here, we compute the three six-scalar subamplitudes (2.2): $A_{s}^{Y}, A_{s}^{X}$ and $A_{s}^{Z}$. To that end, we evaluate the amplitude (3.1) with the correlators

$$
\left\langle V_{\phi^{a_{1}}}^{(0)}\left(z_{1}, k_{1}\right) V_{\phi^{a_{2}}}^{(0)}\left(z_{2}, k_{2}\right) V_{\phi^{a_{3}}}^{(0)}\left(z_{3}, k_{3}\right) V_{\phi^{a_{4}}}^{(0)}\left(z_{4}, k_{4}\right) V_{\phi^{a_{5}}}^{(-1)}\left(z_{5}, k_{5}\right) V_{\phi^{a_{6}}}^{(-1)}\left(z_{6}, k_{6}\right)\right\rangle,
$$

for the helicity configurations $Y, X$ and $Z$. After a straightforward calculation, we obtain

$$
A_{s}=4 \alpha^{\prime} g_{\text {YM }}^{4} L_{5},
$$

with the functions $L_{5} \in\left\{L_{5}^{Y}, L_{5}^{X}, L_{5}^{Z}\right\}$ specific to the three helicity configurations $Y, X$ and $Z$, respectively. Again, we present the integrals $L_{5}$ for the three cases in Appendix A.

\subsection{Four scalars and two vectors}

Finally, we evaluate the three subamplitudes (2.3) involving four scalars and two gauge fields: $A_{g}^{Y}, A_{g}^{X}$ and $A_{g}^{Z}$. To that end, we evaluate the amplitude (3.1) with the correlators

$$
\left\langle V_{\phi^{a_{1}}}^{(0)}\left(z_{1}, k_{1}\right) V_{\phi^{a_{2}}}^{(0)}\left(z_{2}, k_{2}\right) V_{\phi^{a_{3}}}^{(0)}\left(z_{3}, k_{3}\right) V_{\phi^{a_{4}}}^{(0)}\left(z_{4}, k_{4}\right) V_{A^{a_{5}}}^{(-1)}\left(z_{5}, \xi_{5}, k_{5}\right) V_{A^{a_{6}}}^{(-1)}\left(z_{6}, \xi_{6}, k_{6}\right)\right\rangle,
$$

for the helicity configurations $Y, X$ and $Z$. The amplitude $A_{g}^{Y}$ has already been computed in [6]. In fact, all three (partial) amplitudes have a similar form: 


$$
\begin{aligned}
A_{g}= & 8 \alpha^{\prime 2} g_{\mathrm{YM}}^{4}\left[\left(\xi_{5} k_{3}\right)\left(\xi_{6} k_{2}\right) K_{1}+\left(\xi_{5} k_{2}\right)\left(\xi_{6} k_{3}\right) K_{2}+\left(\xi_{5} k_{1}\right)\left(\xi_{6} k_{2}\right) K_{3}\right. \\
& +\left(\xi_{5} k_{1}\right)\left(\xi_{6} k_{3}\right) K_{4}+\left(\xi_{5} k_{2}\right)\left(\xi_{6} k_{1}\right) K_{5}+\left(\xi_{5} k_{3}\right)\left(\xi_{6} k_{1}\right) K_{6} \\
& +\left(\xi_{5} k_{3}\right)\left(\xi_{6} k_{4}\right) K_{7}+\left(\xi_{5} k_{4}\right)\left(\xi_{6} k_{3}\right) K_{8}+\left(\xi_{5} k_{2}\right)\left(\xi_{6} k_{4}\right) K_{9} \\
& \left.+\left(\xi_{5} k_{4}\right)\left(\xi_{6} k_{2}\right) K_{10}+\left(\xi_{5} k_{1}\right)\left(\xi_{6} k_{4}\right) K_{11}+\left(\xi_{5} k_{4}\right)\left(\xi_{6} k_{1}\right) K_{12}+\left(\xi_{5} \xi_{6}\right) K_{13}\right],
\end{aligned}
$$

specified by thirteen functions $K_{i}$ for each configuration $Y, X$ and $Z$. Here, $\xi_{5}$ and $\xi_{6}$ are the gluon polarization vectors. Actually, one also finds $A_{g}^{Y}=A_{g}^{X}$, therefore $K_{i}^{Y}=K_{i}^{X}$. In order to write Eq. (3.15) more explicitly, we choose $k_{6}$ as the reference vector for the (negative) polarization vector of gluon $g_{5}$ and $k_{5}$ as the reference vector for the (positive) polarization vector of gluon $g_{6}$. Then

$$
\xi_{5}^{-} \xi_{6}^{+}=0, \quad\left(\xi_{5}^{-} k_{i}\right)\left(\xi_{6}^{+} k_{j}\right)=-\alpha^{\prime} \frac{[6 i]\langle i 5\rangle[6 j]\langle j 5\rangle}{2 s_{5}},
$$

and the amplitude (3.15) can be rewritten as

$$
\begin{aligned}
A_{g}= & -\frac{4 \alpha^{\prime 3} g_{\mathrm{YM}}^{4}}{s_{5}}\left([6|2| 5\rangle[6|3| 5\rangle G_{1}+[6|1| 5\rangle[6|2| 5\rangle G_{2}+[6|1| 5\rangle[6|3| 5\rangle G_{3}\right. \\
& \left.+[6|3| 5\rangle[6|4| 5\rangle G_{4}+[6|2| 5\rangle[6|4| 5\rangle G_{5}+[6|1| 5\rangle[6|4| 5\rangle G_{6}\right),
\end{aligned}
$$

where $G_{1}=K_{1}+K_{2}, G_{2}=K_{3}+K_{5}, G_{3}=K_{4}+K_{6}, G_{4}=K_{7}+K_{8}, G_{5}=K_{9}+K_{10}$ and $G_{6}=K_{11}+K_{12}$. The integral representations of all these functions are given in Appendix A.

\section{Six-gluon NMHV amplitudes}

After computing all auxiliary amplitudes, we are now in a position to write down the sixgluon amplitudes. The result is obtained by substituting Eqs. (3.10), (3.13) and (3.17) into the r.h.s. of SUSY relations (2.7). We could leave this result as it is, however there are at least two good reasons for trying to combine all contributions into a more compact form. First, although for each helicity configuration, the amplitude depends on ten functions $G, H$ and $L_{5}$, we know that only $(N-3) !=6$ of them are independent [3-5]. Indeed, by using techniques developed in [3], it is possible to find relations between these functions. In what follows, we will combine the ten integrals $G, H$ and $L_{5}$ into a set of six functions $N_{i}$, for each helicity configuration. Second, the kinematic factors appearing in auxiliary amplitudes are related, therefore they can be combined to a form involving fewer kinematic factors. This is highly desirable for many reasons, especially for the comparison of the $\alpha^{\prime}=0$ limit with the well-known QCD amplitudes. The six functions $N_{i}$ will appear naturally in this context. In QCD, six-gluon NMHV amplitudes were first calculated in [14], and later recast in an elegant form in Refs. [10,19]. ${ }^{5}$

The basic relations that allow combining various contributions on the r.h.s. of Eq. (2.7) are:

$$
Y^{2}[16]+[15][6|Y| 5\rangle=-[12][6|Y| 2\rangle,
$$

and its variations obtained by applying various permutations of $\{1,2,3,4,5,6\}$ and/or complex conjugation. Eq. (4.1) follows from Schouten's identity and momentum conservation. The goal is to rewrite our results in a form similar to QCD amplitudes collected in Eq. (5.28) and Table 4 of Ref. [10]. Below, we list the amplitudes obtained by manipulating kinematic factors on the r.h.s. of Eq. (2.7), for the three helicity configurations separately.

\footnotetext{
5 For more recent work on NMHV amplitudes, see, e.g., Refs. [20-24].
} 


\section{1. $Y$-configuration and its $\alpha^{\prime}=0$ limit}

The result will be expressed in terms of the following kinematic variables:

$$
\alpha_{Y}=-\langle 12\rangle[34][6|Y| 5\rangle, \quad \beta_{Y}=\langle 12\rangle[46][3|Y| 5\rangle, \quad \gamma_{Y}=\langle 51\rangle[34][6|Y| 2\rangle,
$$

where $\alpha_{Y}$, already defined in Eq. (2.4), is listed for completeness. Then

$$
\begin{aligned}
A( & \left.g_{1}^{-}, g_{2}^{-}, g_{3}^{+}, g_{4}^{+}, g_{5}^{-}, g_{6}^{+}\right) \\
= & A^{Y} \\
= & \operatorname{Tr}\left(T^{a_{1}} T^{a_{2}} T^{a_{3}} T^{a_{4}} T^{a_{5}} T^{a_{6}}\right) \frac{\left(\sqrt{2} g_{\mathrm{YM}}\right)^{4} \alpha^{\prime}}{s_{5}} \\
& \times\left(N_{1}^{Y} \frac{\alpha_{Y}^{2}}{s_{1}^{2} s_{3}^{2}}+N_{2}^{Y} \frac{\beta_{Y}^{2}}{s_{1}^{2}}+N_{3}^{Y} \frac{\gamma_{Y}^{2}}{s_{3}^{2}}+N_{4}^{Y} \frac{\alpha_{Y} \beta_{Y}}{s_{1}^{2} s_{3}}+N_{5}^{Y} \frac{\alpha_{Y} \gamma_{Y}}{s_{1} s_{3}^{2}}+N_{6}^{Y} \frac{\beta_{Y} \gamma_{Y}}{s_{1} s_{3}}\right),
\end{aligned}
$$

with the functions $N^{Y}$ written below:

$$
\begin{aligned}
N_{1}^{Y}= & -\left(s_{1}+s_{3}+s_{6}-t_{2}-t_{3}\right)\left(s_{1}+s_{3}+s_{4}-t_{1}-t_{3}\right) G_{1}^{Y}-2 s_{5}\left(s_{5}+s_{6}-t_{2}\right) H_{1}^{Y} \\
& -\left(s_{1}+s_{3}+s_{6}-t_{2}-t_{3}\right)\left[\left(s_{5}+s_{6}-t_{2}\right) G_{2}^{Y}-\left(s_{4}+s_{5}-t_{1}\right) G_{5}^{Y}-2 s_{5} H_{2}^{Y}\right] \\
& +\left(s_{1}+s_{3}+s_{4}-t_{1}-t_{3}\right)\left[\left(s_{5}+s_{6}-t_{2}\right) G_{3}^{Y}-\left(s_{4}+s_{5}-t_{1}\right) G_{4}^{Y}-2 s_{5} H_{3}^{Y}\right] \\
& -\left(s_{4}+s_{5}-t_{1}\right)\left(s_{5}+s_{6}-t_{2}\right) G_{6}^{Y}-s_{5} L_{5}^{Y}, \\
N_{2}^{Y}= & -G_{4}^{Y}, \\
N_{3}^{Y}= & -G_{2}^{Y}, \\
N_{4}^{Y}= & \left(s_{1}+s_{3}+s_{6}-t_{2}-t_{3}\right)\left(G_{1}^{Y}-G_{5}^{Y}\right)+\left(s_{1}+s_{3}+2 s_{4}+s_{5}-2 t_{1}-t_{3}\right) G_{4}^{Y} \\
& -\left(s_{5}+s_{6}-t_{2}\right)\left(G_{3}^{Y}-G_{6}^{Y}\right)+2 s_{5} H_{3}^{Y}, \\
N_{5}^{Y}= & \left(s_{1}+s_{3}+s_{4}-t_{1}-t_{3}\right)\left(G_{1}^{Y}-G_{3}^{Y}\right)+\left(s_{1}+s_{3}+s_{5}+2 s_{6}-2 t_{2}-t_{3}\right) G_{2}^{Y} \\
& -\left(s_{4}+s_{5}-t_{1}\right)\left(G_{5}^{Y}-G_{6}^{Y}\right)+2 s_{5}\left(H_{1}^{Y}-H_{2}^{Y}\right), \\
N_{6}^{Y}= & -G_{1}^{Y}+G_{3}^{Y}+G_{5}^{Y}-G_{6}^{Y} .
\end{aligned}
$$

Their low-energy expansions are

$$
\begin{aligned}
& N_{1}^{Y}=\frac{s_{1} s_{3} s_{5}}{s_{4} s_{6} t_{3}}-\zeta(2)\left(s_{1} s_{3}-\frac{s_{1} s_{3} t_{1}}{s_{4}}-\frac{s_{1} s_{3} t_{2}}{s_{6}}+\frac{s_{1} s_{2} s_{3} s_{5}}{s_{4} s_{6}}+\frac{s_{1}^{2} s_{3} s_{5}}{s_{4} t_{3}}+\frac{s_{1} s_{3}^{2} s_{5}}{s_{6} t_{3}}\right)+\cdots, \\
& N_{2}^{Y}=\frac{s_{1}}{s_{2} s_{4} t_{1}}-\zeta(2)\left(\frac{s_{1} s_{6}}{s_{2} s_{4}}+\frac{s_{1}^{2}}{s_{4} t_{1}}+\frac{s_{1} s_{5}}{s_{2} t_{1}}\right)+\cdots, \\
& N_{3}^{Y}=\frac{s_{3}}{s_{2} s_{6} t_{2}}-\zeta(2)\left(\frac{s_{3} s_{4}}{s_{2} s_{6}}+\frac{s_{3} s_{5}}{s_{2} t_{2}}+\frac{s_{3}^{2}}{s_{6} t_{2}}\right)+\cdots, \\
& N_{4}^{Y}=\frac{s_{1} t_{2}}{s_{2} s_{4} s_{6}}-\zeta(2)\left(\frac{s_{1}\left(s_{1}-s_{3}-s_{5}-t_{3}\right)}{s_{4}}-\frac{s_{1} t_{2}}{s_{2}}+\frac{s_{1} t_{1} t_{2}}{s_{2} s_{4}}+\frac{s_{1} t_{2}^{2}}{s_{2} s_{6}}+\frac{s_{1} t_{2} t_{3}}{s_{4} s_{6}}\right)+\cdots, \\
& N_{5}^{Y}=\frac{s_{3} t_{1}}{s_{2} s_{4} s_{6}}+\zeta(2)\left(\frac{s_{3}\left(s_{1}-s_{3}+s_{5}+t_{3}\right)}{s_{6}}+\frac{s_{3} t_{1}}{s_{2}}-\frac{s_{3} t_{1}^{2}}{s_{2} s_{4}}-\frac{s_{3} t_{1} t_{2}}{s_{2} s_{6}}-\frac{s_{3} t_{1} t_{3}}{s_{4} s_{6}}\right)+\cdots, \\
& N_{6}^{Y}=\frac{t_{3}}{s_{2} s_{4} s_{6}}+\zeta(2)\left(\frac{s_{1}+s_{3}-s_{5}}{s_{2}}-\frac{t_{1} t_{3}}{s_{2} s_{4}}-\frac{t_{2} t_{3}}{s_{2} s_{6}}-\frac{t_{3}^{2}}{s_{4} s_{6}}\right)+\cdots,
\end{aligned}
$$


where dots represent terms suppressed by a factor of order $\mathcal{O}\left(\zeta(3) \alpha^{\prime 3}\right)$ with respect to the leading term. The above expansions have been obtained by collecting the expansions of $G$ and $L$ functions listed in Appendix A.

In the $\alpha^{\prime}=0$ limit of the amplitude (4.3), only the leading terms of Eq. (4.5) survive. Then all $\alpha^{\prime}$ factors cancel and Eq. (4.3) agrees with the QCD amplitude written in Eq. (5.28) and Table 4 of Ref. [10]. ${ }^{6}$

\section{2. $X$-configuration and its $\alpha^{\prime}=0$ limit}

The result will be expressed in terms of the following kinematic variables:

$$
\alpha_{X}=-[12]\langle 34\rangle[6|X| 5\rangle, \quad \beta_{X}=[12]\langle 45\rangle[6|X| 3\rangle, \quad \gamma_{X}=[61]\langle 34\rangle[2|X| 5\rangle,
$$

where $\alpha_{X}$, already defined in Eq. (2.4), is listed for completeness. Then

$$
\begin{aligned}
A\left(g_{1}^{+}, g_{2}^{+}, g_{3}^{-}, g_{4}^{-}, g_{5}^{-}, g_{6}^{+}\right) \\
=A^{X} \\
=\operatorname{Tr}\left(T^{a_{1}} T^{a_{2}} T^{a_{3}} T^{a_{4}} T^{a_{5}} T^{a_{6}}\right) \frac{\left(\sqrt{2} g_{\mathrm{YM}}\right)^{4} \alpha^{\prime 5}}{s_{5}} \\
\quad \times\left(N_{1}^{X} \frac{\alpha_{X}^{2}}{s_{1}^{2} s_{3}^{2}}+N_{2}^{X} \frac{\beta_{X}^{2}}{s_{1}^{2}}+N_{3}^{X} \frac{\gamma_{X}^{2}}{s_{3}^{2}}+N_{4}^{X} \frac{\alpha_{X} \beta_{X}}{s_{1}^{2} s_{3}}+N_{5}^{X} \frac{\alpha_{X} \gamma_{X}}{s_{1} s_{3}^{2}}+N_{6}^{X} \frac{\beta_{X} \gamma_{X}}{s_{1} s_{3}}\right),
\end{aligned}
$$

with the functions $N^{X}$ written below:

$$
\begin{aligned}
N_{1}^{X}= & -\left(s_{4}-t_{3}\right)\left(s_{6}-t_{3}\right) G_{1}^{X}-\left(s_{6}-t_{3}\right)\left(s_{6} G_{2}^{X}-s_{4} G_{5}^{X}-2 s_{5} H_{2}^{X}\right) \\
& +\left(s_{4}-t_{3}\right)\left(s_{6} G_{3}^{X}-s_{4} G_{4}^{X}-2 s_{5} H_{3}^{X}\right)-s_{6}\left(s_{4} G_{6}^{X}+2 s_{5} H_{1}^{X}\right)-s_{5} L_{5}^{X}, \\
N_{2}^{X}= & -G_{4}^{X}, \\
N_{3}^{X}= & -G_{2}^{X}, \\
N_{4}^{X}= & -\left(s_{6}-t_{3}\right)\left(G_{1}^{X}-G_{5}^{X}\right)+s_{6}\left(G_{3}^{X}-G_{6}^{X}\right)-\left(2 s_{4}-t_{3}\right) G_{4}^{X}-2 s_{5} H_{3}^{X}, \\
N_{5}^{X}= & -\left(s_{4}-t_{3}\right)\left(G_{1}^{X}-G_{3}^{X}\right)-\left(2 s_{6}-t_{3}\right) G_{2}^{X}+s_{4}\left(G_{5}^{X}-G_{6}^{X}\right)-2 s_{5}\left(H_{1}^{X}-H_{2}^{X}\right), \\
N_{6}^{X}= & -G_{1}^{X}+G_{3}^{X}+G_{5}^{X}-G_{6}^{X} .
\end{aligned}
$$

Their low-energy expansions are

$$
\begin{aligned}
& N_{1}^{X}=-\zeta(2) s_{1} s_{3}+\cdots, \\
& N_{2}^{X}=\frac{s_{1}}{s_{2} s_{4} t_{1}}-\zeta(2)\left(\frac{s_{1} s_{6}}{s_{2} s_{4}}+\frac{s_{1}^{2}}{s_{4} t_{1}}+\frac{s_{1} s_{5}}{s_{2} t_{1}}\right)+\cdots, \\
& N_{3}^{X}=\frac{s_{3}}{s_{2} s_{6} t_{2}}-\zeta(2)\left(\frac{s_{3} s_{4}}{s_{2} s_{6}}+\frac{s_{3} s_{5}}{s_{2} t_{2}}+\frac{s_{3}^{2}}{s_{6} t_{2}}\right)+\cdots, \\
& N_{4}^{X}=\zeta(2)\left(\frac{s_{1} t_{2}}{s_{2}}+\frac{s_{1} t_{3}}{s_{4}}\right)+\cdots,
\end{aligned}
$$

6 In order to compare, a cyclic permutation $\{1,2,3,4,5,6\} \rightarrow\{3,4,5,6,1,2\}$ must be performed on the result of Ref. [10]. 


$$
\begin{aligned}
& N_{5}^{X}=\zeta(2)\left(\frac{s_{3} t_{1}}{s_{2}}+\frac{s_{3} t_{3}}{s_{6}}\right)+\cdots, \\
& N_{6}^{X}=\frac{t_{3}}{s_{2} s_{4} s_{6}}+\zeta(2)\left(\frac{s_{1}+s_{3}-s_{5}}{s_{2}}-\frac{t_{1} t_{3}}{s_{2} s_{4}}-\frac{t_{2} t_{3}}{s_{2} s_{6}}-\frac{t_{3}^{2}}{s_{4} s_{6}}\right)+\cdots,
\end{aligned}
$$

where dots represent terms suppressed by a factor of order $\mathcal{O}\left(\zeta(3) \alpha^{\prime 3}\right)$ with respect to the leading (QCD) contribution. The $\alpha^{\prime}=0$ limit of the amplitude (4.7) agrees with Ref. [10]. ${ }^{7}$ Note, in particular, that all terms multiplying $\alpha_{X}$ disappear in this limit.

\subsection{Z-configuration and its $\alpha^{\prime}=0$ limit}

The result will be expressed in terms of the following kinematic variables:

$$
\alpha_{Z}=-\langle 13\rangle[24][6|Z| 5\rangle, \quad \beta_{Z}=\langle 13\rangle[46][2|Z| 5\rangle, \quad \gamma_{Z}=\langle 51\rangle[24][6|Z| 3\rangle,
$$

where $\alpha_{Z}$, already defined in Eq. (2.4), is listed for completeness. Then

$$
\begin{aligned}
A\left(g_{1}^{-}, g_{2}^{+}, g_{3}^{-}, g_{4}^{+}, g_{5}^{-}, g_{6}^{+}\right) & =A^{Z} \\
= & \operatorname{Tr}\left(T^{a_{1}} T^{a_{2}} T^{a_{3}} T^{a_{4}} T^{a_{5}} T^{a_{6}}\right) \frac{\left(\sqrt{2} g_{\mathrm{YM}}\right)^{4} \alpha^{\prime 5}}{s_{5}} \\
& \times\left(N_{1}^{Z} \frac{\alpha_{Z}^{2}}{s_{13}^{2} s_{24}^{2}}+N_{2}^{Z} \frac{\beta_{Z}^{2}}{s_{13}^{2}}+N_{3}^{Z} \frac{\gamma_{Z}^{2}}{s_{24}^{2}}+N_{4}^{Z} \frac{\alpha_{Z} \beta_{Z}}{s_{13}^{2} s_{24}}+N_{5}^{Z} \frac{\alpha_{Z} \gamma_{Z}}{s_{13} s_{24}^{2}}+N_{6}^{Z} \frac{\beta_{Z} \gamma_{Z}}{s_{13} s_{24}}\right),
\end{aligned}
$$

with the functions $N^{Z}$ written below:

$$
\begin{aligned}
N_{1}^{Z}= & -\left(s_{1}+s_{2}+s_{3}+s_{6}-t_{2}-t_{3}\right)\left(s_{1}+s_{2}+s_{3}+s_{4}-t_{1}-t_{3}\right) G_{1}^{Z} \\
& -\left(s_{1}+s_{2}+s_{3}+s_{6}-t_{2}-t_{3}\right)\left[\left(s_{5}+s_{6}-t_{2}\right) G_{2}^{Z}-\left(s_{4}+s_{5}-t_{1}\right) G_{5}^{Z}-2 s_{5} H_{2}^{Z}\right] \\
& +\left(s_{1}+s_{2}+s_{3}+s_{4}-t_{1}-t_{3}\right)\left[\left(s_{5}+s_{6}-t_{2}\right) G_{3}^{Z}-\left(s_{4}+s_{5}-t_{1}\right) G_{4}^{Z}-2 s_{5} H_{3}^{Z}\right] \\
& -\left(s_{4}+s_{5}-t_{1}\right)\left(s_{5}+s_{6}-t_{2}\right) G_{6}^{Z}-2 s_{5}\left(s_{5}+s_{6}-t_{2}\right) H_{1}^{Z}-s_{5} L_{5}^{Z}, \\
N_{2}^{Z}= & -G_{5}^{Z}, \\
N_{3}^{Z}= & -G_{3}^{Z}, \\
N_{4}^{Z}= & -\left(s_{1}+s_{2}+s_{3}+s_{4}-t_{1}-t_{3}\right)\left(G_{1}^{Z}-G_{4}^{Z}+G_{5}^{Z}\right)-\left(s_{5}+s_{6}-t_{2}\right)\left(G_{2}^{Z}-G_{5}^{Z}-G_{6}^{Z}\right) \\
& +2\left(s_{4}-s_{6}-t_{1}+t_{2}\right) G_{5}^{Z}+2 s_{5} H_{2}^{Z}, \\
N_{5}^{Z}= & -\left(s_{1}+s_{2}+s_{3}+s_{6}-t_{2}-t_{3}\right)\left(G_{1}^{Z}-G_{2}^{Z}+G_{3}^{Z}\right)-\left(s_{4}+s_{5}-t_{1}\right)\left(G_{3}^{Z}+G_{4}^{Z}-G_{6}^{Z}\right) \\
& +2\left(s_{5}+s_{6}-t_{2}\right) G_{3}^{Z}+2 s_{5}\left(H_{1}^{Z}-H_{3}^{Z}\right), \\
N_{6}^{Z}= & -G_{1}^{Z}+G_{2}^{Z}+G_{4}^{Z}-G_{6}^{Z} .
\end{aligned}
$$

The low-energy expansions of these functions are much more complicated in the two previous cases:

$$
N_{1}^{Z}=\left(s_{1}+s_{2}-t_{1}\right)\left(s_{2}+s_{3}-t_{2}\right)\left(\frac{s_{2}}{s_{1} s_{3} t_{3}}+\frac{s_{5}}{s_{4} s_{6} t_{3}}+\frac{t_{1}}{s_{1} s_{4} t_{3}}+\frac{t_{2}}{s_{3} s_{6} t_{3}}\right)+\cdots,
$$

\footnotetext{
7 In this case, a cyclic permutation $\{1,2,3,4,5,6\} \rightarrow\{6,1,2,3,4,5\}$ must be performed on the result of Ref. [10].
} 


$$
\begin{aligned}
N_{2}^{Z}= & \left(s_{1}+s_{2}-t_{1}\right)\left(\frac{1}{s_{1} s_{3} t_{3}}+\frac{1}{s_{1} s_{4} t_{3}}+\frac{1}{s_{1} s_{4} t_{1}}+\frac{1}{s_{2} s_{4} t_{1}}\right)+\cdots, \\
N_{3}^{Z}= & \left(s_{2}+s_{3}-t_{2}\right)\left(\frac{1}{s_{1} s_{3} t_{3}}+\frac{1}{s_{3} s_{6} t_{3}}+\frac{1}{s_{2} s_{6} t_{2}}+\frac{1}{s_{3} s_{6} t_{2}}\right)+\cdots, \\
N_{4}^{Z}= & \left(s_{1}+s_{2}-t_{1}\right)\left(\frac{2 s_{2}}{s_{1} s_{3} t_{3}}+\frac{s_{2}}{s_{1} s_{4} t_{3}}+\frac{1}{s_{1} s_{4}}-\frac{s_{5}}{s_{1} s_{4} t_{3}}-\frac{s_{6}}{s_{1} s_{4} t_{3}}+\frac{t_{1}}{s_{1} s_{4} t_{3}}-\frac{t_{2}}{s_{1} s_{3} t_{3}}\right. \\
& \left.+\frac{t_{2}}{s_{3} s_{6} t_{3}}+\frac{t_{2}}{s_{4} s_{6} t_{3}}+\frac{1}{s_{1} t_{3}}+\frac{s_{3}}{s_{1} s_{4} t_{3}}-\frac{1}{s_{4} t_{3}}+\frac{t_{2}}{s_{4} s_{6} s_{2}}\right)+\cdots, \\
N_{5}^{Z}= & \left(s_{2}+s_{3}-t_{2}\right)\left(\frac{s_{1}}{s_{3} s_{6} t_{3}}+\frac{t_{1}}{s_{2} s_{4} s_{6}}+\frac{1}{s_{3} s_{6}}+\frac{t_{1}}{s_{4} s_{6} t_{3}}+\frac{t_{2}}{s_{3} s_{6} t_{3}}+\frac{1}{s_{3} t_{3}}-\frac{s_{4}}{s_{3} s_{6} t_{3}}\right. \\
& \left.-\frac{s_{5}}{s_{3} s_{6} t_{3}}+\frac{s_{2}}{s_{3} s_{6} t_{3}}-\frac{1}{s_{6} t_{3}}-\frac{t_{1}}{s_{3} t_{3} s_{1}}+\frac{t_{1}}{s_{4} t_{3} s_{1}}+\frac{2 s_{2}}{s_{3} t_{3} s_{1}}\right)+\cdots, \\
& \frac{2 s_{2}}{s_{2}}+\frac{s_{2}}{s_{1} s_{4} t_{3}}+\frac{s_{2}}{s_{3} s_{6} t_{3}}+\frac{s_{2}}{s_{4} s_{6} t_{3}}+\frac{1}{s_{1} s_{4}}+\frac{1}{s_{3} s_{6}}+\frac{2}{s_{4} s_{6}}+\frac{s_{5}}{s_{1} s_{3} t_{3}}-\frac{s_{6}}{s_{1} s_{4} t_{3}} \\
& -\frac{t_{1}}{s_{1} s_{3} t_{3}}-\frac{t_{2}}{s_{1} s_{3} t_{3}}-\frac{1}{s_{4} t_{3}}-\frac{s_{4}}{s_{3} s_{6} t_{3}}-\frac{1}{s_{6} t_{3}}+\frac{t_{3}}{s_{4} s_{6} s_{2}}-\frac{1}{s_{4} s_{2}}-\frac{1}{s_{6} s_{2}}+\cdots,
\end{aligned}
$$

where dots represent terms suppressed by a factor of order $\mathcal{O}\left(\zeta(2) \alpha^{\prime 2}\right.$ ) with respect to the leading (QCD) contributions to the amplitude. The order $\zeta(2) \alpha^{\prime 2}$ is presented in Appendix B.

For this helicity configuration, the comparison of the $\alpha^{\prime}=0$ limit with QCD is a highly nontrivial and tedious exercise in spinor algebra which, fortunately, has a happy end. Most likely, another SUSY relation would be more efficient in handling this case.

More details on the functions $N_{i}$ are given in Appendix B.

\section{Summary and outlook}

Together with the MHV amplitudes presented in Refs. [4-6], the NMHV amplitudes presented in this work provide the complete six-gluon disk amplitude. As expected, the NMHV case is considerably more complex than MHV. Six gluons are still manageable (as well as sevengluon MHVs [6]), but clearly more efficient techniques need to be developed for handling larger numbers of external gluons. To that end, some type of recursion relations should be constructed, similar to Berends-Giele relations [25] in QCD and/or to the so-called MHV or recursive rules $[21,26,27]$. This is quite an involved task: all string excitations propagate in intermediate channels of the disk diagram, therefore a part of the problem is to extend string propagation off mass-shell.

In addition to possible phenomenological applications of our results already stressed in the Introduction, we should point out that the complete six-gluon string amplitude, together with the previously obtained five- and four-gluon amplitudes (summarized in Refs. [4,5]), provide all information necessary for constructing the non-Abelian Born-Infeld action up to the order $\mathcal{O}\left(\alpha^{\prime 4} F^{6}\right)$ in the gauge field strength $F$. Furthermore, a direct comparison of type I disk amplitudes with two-loop heterotic amplitudes-a non-trivial test of type I-heterotic duality [28]-becomes now possible.

It is interesting that multi-gluon disk amplitudes exhibit transcendentality behavior in their low-energy $\alpha^{\prime}$-expansions. Each power $\alpha^{\prime n}$ comes with the factor $\zeta(n)$, a product of zeta functions or multiple zeta values having transcendentality degree $n$. 


\section{Acknowledgements}

We are grateful to Lance Dixon for a very useful correspondence that allowed improving the original version of this paper. This work is supported in part by the European Commission under Project MRTN-CT-2004-005104. The research of T.R.T. is supported in part by the US National Science Foundation Grant PHY-0600304. S.S. would like to thank the Galileo Galilei Institute for Theoretical Physics in Firenze and the Albert-Einstein-Institut in Potsdam for hospitality and INFN and AEI for partial support during completion of this work. He is grateful to Hermann Nicolai for inviting him to the AEI. T.R.T. thanks Max-Planck-Institute in Munich and High Energy Theory Group at Harvard University for their kind hospitality. He is deeply indebted to Dieter Lüst for a timely invitation to Munich. Any opinions, findings, and conclusions or recommendations expressed in this material are those of the authors and do not necessarily reflect the views of the National Science Foundation.

\section{Appendix A. Hypergeometric functions $L_{i}, G_{j}$ and their $\alpha^{\prime}$ expansions}

In this appendix, we collect the hypergeometric functions $L_{i}$ and $G_{i}$ describing the auxiliary amplitudes (3.9), (3.13) and (3.17).

\section{A.1. Helicity configuration $Y$}

The functions $L_{i}$ entering (3.9) are:

$$
\begin{aligned}
& L_{1}^{Y}=s_{23} L_{4}^{Y}-\int_{0}^{1} d x \int_{0}^{1} d y \int_{0}^{1} d z\left(s_{23}+\frac{1-s_{23}}{x}\right) \frac{\mathcal{I}(x, y, z)}{x y z(1-y)(1-z)}, \\
& L_{2}^{Y}=-s_{13} L_{4}^{Y}-\int_{0}^{1} d x \int_{0}^{1} d y \int_{0}^{1} d z\left(1-s_{13}+\frac{s_{13}}{x}\right) \frac{\mathcal{I}(x, y, z)}{(1-y)(1-z)}, \\
& L_{3}^{Y}=s_{12} L_{4}^{Y}+s_{12} \int_{0}^{1} d x \int_{0}^{1} d y \int_{0}^{1} d z \frac{1-x}{1-z} \frac{\mathcal{I}(x, y, z)}{x(1-y)(1-x y)}, \\
& L_{4}^{Y}=\int_{0}^{1} d x \int_{0}^{1} d y \int_{0}^{1} d z \frac{(1-x) \mathcal{I}(x, y, z)}{x z(1-y)(1-x y)} .
\end{aligned}
$$

Their low-energy expansions are:

$$
\begin{aligned}
& L_{1}^{Y}=-\frac{1}{s_{2} s_{4}}-\frac{1}{s_{2} s_{5}}-\frac{1}{s_{2} s_{6}}+\frac{1}{s_{4} s_{6}}+\frac{s_{1}}{s_{2} s_{4} t_{1}}+\frac{s_{1}}{s_{2} s_{5} t_{1}}+\frac{s_{3}}{s_{2} s_{5} t_{2}}+\frac{s_{3}}{s_{2} s_{6} t_{2}}+\frac{t_{3}}{s_{2} s_{4} s_{6}}+\cdots, \\
& L_{2}^{Y}=-\frac{1}{s_{2} s_{4}}-\frac{1}{s_{2} s_{5}}+\frac{1}{s_{4} s_{6}}+\frac{s_{1}}{s_{2} s_{4} s_{6}}+\frac{s_{1}}{s_{2} s_{4} t_{1}}+\frac{s_{1}}{s_{2} s_{5} t_{1}}-\frac{t_{1}}{s_{2} s_{4} s_{6}}+\cdots, \\
& L_{3}^{Y}=\frac{s_{1}}{s_{2} s_{4} s_{6}}+\frac{s_{1}}{s_{2} s_{4} t_{1}}+\frac{s_{1}}{s_{2} s_{5} t_{1}}+\cdots, \quad L_{4}^{Y}=\frac{1}{s_{2} s_{4} s_{6}}+\cdots .
\end{aligned}
$$


The function $L_{5}$ entering (3.13) is:

$$
\begin{aligned}
L_{5}^{Y}= & -\int_{0}^{1} d x \int_{0}^{1} d y \int_{0}^{1} d z\left[\left(1-s_{13} \frac{1-x y z}{z(1-x y)}\right)\left(1-s_{24} \frac{1-y z}{1-y}\right)\right. \\
& +\frac{1}{x^{2}}\left(1-s_{14} \frac{1-y z}{z(1-y)}\right)\left(1-s_{23} \frac{1-x y z}{1-x y}\right) \\
& \left.+\frac{(1-y z)(1-x y z)}{x z(1-y)(1-x y)}\left(s_{12} s_{34}-s_{14} s_{23}-s_{13} s_{24}\right)\right] \frac{\mathcal{I}(x, y, z)}{y(1-z)^{2}} .
\end{aligned}
$$

It has the following $\alpha^{\prime}$-expansion:

$$
\begin{aligned}
L_{5}^{Y}= & \frac{1}{s_{2} s_{4}}\left(s_{1}-s_{3}+s_{5}-\frac{s_{1} s_{5}}{t_{1}}-t_{1}\right)+\frac{1}{s_{2} s_{6}}\left(-s_{1}+s_{3}+s_{5}-\frac{s_{3} s_{5}}{t_{2}}-t_{2}\right) \\
& +\frac{1}{s_{4} s_{6}}\left(s_{1}+s_{3}-s_{5}-\frac{s_{1} s_{3}}{t_{3}}-t_{3}\right)-\frac{1}{s_{2} s_{4} s_{6}}\left(s_{3} t_{1}+s_{1} t_{2}+s_{5} t_{3}-t_{1} t_{2}-t_{1} t_{3}-t_{2} t_{3}\right) \\
& -\frac{1}{s_{2} s_{5}}\left(\frac{s_{1} s_{4}}{t_{1}}+\frac{s_{3} s_{6}}{t_{2}}-t_{3}\right)+\frac{2}{s_{2}}\left(1-\frac{s_{1}}{t_{1}}-\frac{s_{3}}{t_{2}}\right)+\cdots .
\end{aligned}
$$

The functions $G_{i}$ entering (3.17) are:

$$
\begin{aligned}
& G_{1}^{Y}=\int_{0}^{1} d x \int_{0}^{1} d y \int_{0}^{1} d z\left(\frac{1-s_{14}}{x}+s_{14}\right) \frac{x y \mathcal{I}(x, y, z)}{(1-x y)(1-x y z)}, \\
& G_{2}^{Y}=-s_{34} \int_{0}^{1} d x \int_{0}^{1} d y \int_{0}^{1} d z \frac{\mathcal{I}(x, y, z)}{x y z}, \\
& G_{3}^{Y}=\int_{0}^{1} d x \int_{0}^{1} d y \int_{0}^{1} d z\left(1-s_{24}+\frac{s_{24}}{x}\right) \frac{\mathcal{I}(x, y, z)}{y z(1-x y)(1-x y z)}, \\
& G_{4}^{Y}=-s_{12} \int_{0}^{1} d x \int_{0}^{1} d y \int_{0}^{1} d z \frac{y(1-x)^{2} \mathcal{I}(x, y, z)}{x(1-y)(1-x y)(1-y z)(1-x y z)}, \\
& G_{5}^{Y}=\int_{0}^{1} d x \int_{0}^{1} d y \int_{0}^{1} d z\left(1-s_{13}+\frac{s_{13}}{x}\right) \frac{y \mathcal{I}(x, y, z)}{(1-y)(1-y z)}, \\
& G_{6}^{Y}=\int_{0}^{1} d x \int_{0}^{1} d y \int_{0}^{1} d z\left(\frac{1-s_{23}}{x}+s_{23}\right) \frac{\mathcal{I}(x, y, z)}{x y z(1-y)(1-y z)} .
\end{aligned}
$$

Their low-energy expansions are

$$
\begin{aligned}
& G_{1}^{Y}=\zeta(2)+\cdots, \quad G_{2}^{Y}=-\frac{s_{3}}{s_{2} s_{6} t_{2}}+\zeta(2)\left(\frac{s_{3} s_{4}}{s_{2} s_{6}}+\frac{s_{3} s_{5}}{s_{2} t_{2}}+\frac{s_{3}^{2}}{s_{6} t_{2}}\right)+\cdots, \\
& G_{3}^{Y}=\frac{1}{s_{2} s_{6}}-\frac{s_{3}}{s_{2} s_{6} t_{2}}+\zeta(2)\left(1-\frac{s_{5}}{s_{2}}-\frac{s_{3}}{s_{6}}-\frac{t_{3}}{s_{6}}+\frac{s_{3} s_{4}}{s_{2} s_{6}}+\frac{s_{3} s_{5}}{s_{2} t_{2}}+\frac{s_{3}^{2}}{s_{6} t_{2}}-\frac{s_{4} t_{2}}{s_{2} s_{6}}\right)+\cdots,
\end{aligned}
$$




$$
\begin{aligned}
G_{4}^{Y}= & -\frac{s_{1}}{s_{2} s_{4} t_{1}}+\zeta(2)\left(\frac{s_{1} s_{6}}{s_{2} s_{4}}+\frac{s_{1}^{2}}{s_{4} t_{1}}+\frac{s_{1} s_{5}}{s_{2} t_{1}}\right)+\cdots, \\
G_{5}^{Y}= & \frac{1}{s_{2} s_{4}}-\frac{s_{1}}{s_{2} s_{4} t_{1}}+\zeta(2)\left(1-\frac{s_{1}}{s_{4}}-\frac{s_{5}}{s_{2}}-\frac{t_{3}}{s_{4}}+\frac{s_{1} s_{6}}{s_{2} s_{4}}+\frac{s_{1} s_{5}}{s_{2} t_{1}}+\frac{s_{1}^{2}}{s_{4} t_{1}}-\frac{s_{6} t_{1}}{s_{2} s_{4}}\right)+\cdots, \\
G_{6}^{Y}= & \frac{1}{s_{2} s_{4}}+\frac{1}{s_{2} s_{6}}-\frac{s_{1}}{s_{2} s_{4} t_{1}}-\frac{t_{3}}{s_{2} s_{4} s_{6}}-\frac{s_{3}}{s_{2} s_{6} t_{2}}+\zeta(2)\left(1-\frac{s_{1}}{s_{2}}-\frac{s_{3}}{s_{2}}-\frac{s_{5}}{s_{2}}-\frac{s_{1}}{s_{4}}-\frac{t_{3}}{s_{4}}\right. \\
& -\frac{s_{3}}{s_{6}}-\frac{t_{3}}{s_{6}}+\frac{s_{1}^{2}}{s_{4} t_{1}}+\frac{s_{1} s_{6}}{s_{2} s_{4}}+\frac{s_{3} s_{4}}{s_{2} s_{6}}+\frac{s_{3} s_{5}}{s_{2} t_{2}}+\frac{s_{1} s_{5}}{s_{2} t_{1}}+\frac{t_{3}^{2}}{s_{4} s_{6}}-\frac{s_{6} t_{1}}{s_{2} s_{4}}-\frac{s_{4} t_{2}}{s_{2} s_{6}} \\
& \left.+\frac{t_{1} t_{3}}{s_{2} s_{4}}+\frac{t_{2} t_{3}}{s_{2} s_{6}}+\frac{s_{3}^{2}}{s_{6} t_{2}}\right)+\cdots,
\end{aligned}
$$

where dots represent terms suppressed by a factor of $\operatorname{order} \mathcal{O}\left(\zeta(3) \alpha^{\prime 3}\right)$ with respect to the leading (QCD) contribution.

\section{A.2. Helicity configuration $X$}

The functions $L_{i}$ entering (3.9) are:

$$
\begin{aligned}
& L_{1}^{X}=-\int_{0}^{1} d x \int_{0}^{1} d y \int_{0}^{1} d z\left(s_{23}+\frac{1-s_{23}}{x}\right) \frac{\mathcal{I}(x, y, z)}{x y(1-z)(1-y z)}, \\
& L_{2}^{X}=-\int_{0}^{1} d x \int_{0}^{1} d y \int_{0}^{1} d z\left(1-s_{13}+\frac{s_{13}}{x}\right) \frac{\mathcal{I}(x, y, z)}{(1-z)(1-y z)}, \\
& L_{3}^{X}=s_{12} \int_{0}^{1} d x \int_{0}^{1} d y \int_{0}^{1} d z \frac{1-x}{1-z} \frac{\mathcal{I}(x, y, z)}{x(1-y z)(1-x y z)}, \\
& L_{4}^{X}=\int_{0}^{1} d x \int_{0}^{1} d y \int_{0}^{1} d z \frac{(1-x) \mathcal{I}(x, y, z)}{x(1-y z)(1-x y z)} .
\end{aligned}
$$

Their low-energy expansions are:

$$
\begin{aligned}
& L_{1}^{X}=-\frac{1}{s_{2} s_{5}}+\frac{s_{1}}{s_{2} s_{5} t_{1}}+\frac{s_{3}}{s_{2} s_{5} t_{2}}+\cdots, \quad L_{2}^{X}=-\frac{1}{s_{2} s_{5}}+\frac{s_{1}}{s_{2} s_{5} t_{1}}+\cdots, \\
& L_{3}^{X}=\frac{s_{1}}{s_{2} s_{5} t_{1}}+\cdots, \quad L_{4}^{X}=\cdots .
\end{aligned}
$$

The function $L_{5}$ entering (3.13) is

$$
\begin{aligned}
L_{5}^{X}= & -\int_{0}^{1} d x \int_{0}^{1} d y \int_{0}^{1} d z\left[\left(1-s_{13} \frac{z(1-x y)}{(1-x y z)}\right)\left(1-s_{24} \frac{1-y}{1-y z}\right)\right. \\
& +\frac{1}{x^{2}}\left(1-s_{14} \frac{(1-y) z}{1-y z}\right)\left(1-s_{23} \frac{1-x y}{1-x y z}\right)
\end{aligned}
$$




$$
\left.+\frac{(1-y)(1-x y) z}{x(1-y z)(1-x y z)}\left(s_{12} s_{34}-s_{14} s_{23}-s_{13} s_{24}\right)\right] \frac{\mathcal{I}(x, y, z)}{y(1-z)^{2}} .
$$

It has the following $\alpha^{\prime}$-expansion:

$$
L_{5}^{X}=\frac{t_{3}}{s_{2} s_{5}}-\frac{s_{1} s_{4}}{s_{2} s_{5} t_{1}}-\frac{s_{3} s_{6}}{s_{2} s_{5} t_{2}}+\cdots
$$

where dots represent terms suppressed by a factor of order $\mathcal{O}\left(\zeta(2) \alpha^{\prime 2}\right)$ with respect to the leading (QCD) contribution.

As already mentioned before $A_{g}^{X}=A_{g}^{Y}$, therefore the functions $G_{i}^{X}=G_{i}^{Y}$, see Eq. (A.5).

\section{A.3. Helicity configuration $Z$}

The functions $L_{i}$ entering (3.9) are:

$$
\begin{aligned}
& L_{1}^{Z}=-\int_{0}^{1} d x \int_{0}^{1} d y \int_{0}^{1} d z\left(1-\frac{s_{23}}{1-x}\right) \frac{\mathcal{I}(x, y, z)}{x^{2} y z(1-y)(1-z)}, \\
& L_{2}^{Z}=-s_{13} \int_{0}^{1} d x \int_{0}^{1} d y \int_{0}^{1} d z \frac{\mathcal{I}(x, y, z)}{x z(1-x)(1-y)(1-z)}, \\
& L_{3}^{Z}=-\int_{0}^{1} d x \int_{0}^{1} d y \int_{0}^{1} d z\left(1-\frac{s_{12}}{x z}\right) \frac{\mathcal{I}(x, y, z)}{(1-x)(1-y)(1-z)(1-x y z)}, \\
& L_{4}^{Z}=\int_{0}^{1} d x \int_{0}^{1} d y \int_{0}^{1} d z \frac{\mathcal{I}(x, y, z)}{x z(1-x)(1-y)(1-x y z)} .
\end{aligned}
$$

Their low-energy expansions are:

$$
\begin{aligned}
L_{1}^{Z}= & \frac{s_{1}}{s_{2} s_{4} t_{1}}+\frac{s_{1}}{s_{2} s_{5} t_{1}}+\frac{t_{3}}{s_{2} s_{4} s_{6}}+\frac{s_{3}}{s_{2} s_{5} t_{2}}+\frac{s_{2}}{s_{3} s_{5} t_{2}}+\frac{s_{3}}{s_{2} s_{6} t_{2}}+\frac{s_{2}}{s_{3} s_{6} t_{2}}+\frac{s_{2}}{s_{3} s_{6} t_{3}} \\
& +\frac{s_{2}}{s_{4} s_{6} t_{3}}+\frac{s_{2}}{s_{1} s_{3} s_{5}}+\frac{s_{2}}{s_{1} s_{4} t_{1}}+\frac{s_{2}}{s_{1} s_{5} t_{1}}+\frac{s_{2}}{s_{1} s_{3} t_{3}}+\frac{s_{2}}{s_{1} s_{4} t_{3}}-\frac{1}{s_{2} s_{4}}-\frac{1}{s_{2} s_{5}}-\frac{1}{s_{2} s_{6}} \\
& +\frac{2}{s_{4} s_{6}}+\frac{2}{s_{4} t_{1}}+\frac{2}{s_{5} t_{1}}+\frac{2}{s_{5} t_{2}}+\frac{2}{s_{6} t_{2}}+\cdots, \\
L_{2}^{Z}= & \left(s_{1}+s_{2}-t_{1}\right)\left(\frac{1}{s_{2} s_{4} s_{6}}+\frac{1}{s_{2} s_{4} t_{1}}+\frac{1}{s_{1} s_{5} t_{1}}+\frac{1}{s_{2} s_{5} t_{1}}+\frac{1}{s_{3} s_{6} t_{3}}+\frac{1}{s_{4} s_{6} t_{3}}+\frac{1}{s_{1} s_{3} s_{5}}\right. \\
& \left.+\frac{1}{s_{1} s_{3} t_{3}}+\frac{1}{s_{1} s_{4} t_{1}}+\frac{1}{s_{1} s_{4} t_{3}}\right)+\cdots, \\
L_{3}^{Z}= & \frac{s_{1}}{s_{2} s_{4} s_{6}}+\frac{s_{1}}{s_{2} s_{4} t_{1}}+\frac{s_{1}}{s_{2} s_{5} t_{1}}+\frac{s_{1}}{s_{3} s_{6} t_{3}}+\frac{s_{1}}{s_{4} s_{6} t_{3}}+\frac{t_{2}}{s_{1} s_{3} s_{5}}+\frac{s_{2}}{s_{1} s_{4} t_{1}}+\frac{s_{2}}{s_{1} s_{5} t_{1}}+\frac{s_{6}}{s_{1} s_{3} t_{3}} \\
& +\frac{s_{6}}{s_{1} s_{4} t_{3}}-\frac{1}{s_{1} s_{5}}+\frac{1}{s_{3} s_{5}}-\frac{1}{s_{1} s_{3}}-\frac{1}{s_{1} s_{4}}+\frac{2}{s_{4} t_{1}}+\frac{2}{s_{5} t_{1}}+\frac{2}{s_{3} t_{3}}+\frac{2}{s_{4} t_{3}}+\cdots, \\
L_{4}^{Z}= & \frac{1}{s_{2} s_{4} s_{6}}+\frac{1}{s_{1} s_{3} t_{3}}+\frac{1}{s_{1} s_{4} t_{3}}+\frac{1}{s_{3} s_{6} t_{3}}+\frac{1}{s_{4} s_{6} t_{3}}+\cdots \cdot
\end{aligned}
$$


The function $L_{5}$ entering (3.13) is

$$
\begin{aligned}
L_{5}^{Z}= & -\int_{0}^{1} d x \int_{0}^{1} d y \int_{0}^{1} d z\left[\frac{1}{(1-x)^{2}}\left(1-\frac{s_{12}}{z}\right)\left(1-s_{34} \frac{(1-x y)(1-y z)}{(1-y)(1-x y z)}\right)\right. \\
& +\frac{1}{x^{2}}\left(1-s_{14} \frac{1-y z}{(1-y) z}\right)\left(1-s_{23} \frac{1-x y}{1-x y z}\right) \\
& \left.+\frac{(1-x y)(1-y z)}{x z(1-x)(1-y)(1-x y z)}\left(s_{12} s_{34}+s_{14} s_{23}-s_{13} s_{24}\right)\right] \frac{\mathcal{I}(x, y, z)}{y(1-z)^{2}} .
\end{aligned}
$$

It has the following expansion:

$$
\begin{aligned}
L_{5}^{Z}= & \frac{1}{s_{2} s_{4}}\left(s_{1}-s_{3}+s_{5}-\frac{s_{1} s_{5}}{t_{1}}-t_{1}\right)+\frac{1}{s_{2} s_{6}}\left(-s_{1}+s_{3}+s_{5}-\frac{s_{3} s_{5}}{t_{2}}-t_{2}\right) \\
& +\frac{1}{s_{4} s_{6}}\left(s_{1}+s_{3}-s_{5}-\frac{s_{1} s_{3}}{t_{3}}-t_{3}\right)-\frac{1}{s_{2} s_{4} s_{6}}\left(s_{3} t_{1}+s_{1} t_{2}+s_{5} t_{3}-t_{1} t_{2}-t_{1} t_{3}-t_{2} t_{3}\right) \\
& +\frac{1}{s_{3} s_{5}}\left(s_{2}-s_{4}+s_{6}-\frac{s_{2} s_{6}}{t_{2}}-t_{2}\right)+\frac{1}{s_{1} s_{3}}\left(-s_{2}+s_{4}+s_{6}-\frac{s_{4} s_{6}}{t_{3}}-t_{3}\right) \\
& +\frac{1}{s_{1} s_{5}}\left(s_{2}+s_{4}-s_{6}-\frac{s_{2} s_{4}}{t_{1}}-t_{1}\right)-\frac{1}{s_{1} s_{3} s_{5}}\left(s_{4} t_{2}+s_{2} t_{3}+s_{6} t_{1}-t_{2} t_{3}-t_{1} t_{2}-t_{1} t_{3}\right) \\
& -\frac{1}{s_{2} s_{5}}\left(\frac{s_{1} s_{4}}{t_{1}}+\frac{s_{3} s_{6}}{t_{2}}-t_{3}\right)-\frac{1}{s_{3} s_{6}}\left(\frac{s_{2} s_{5}}{t_{2}}+\frac{s_{1} s_{4}}{t_{3}}-t_{1}\right)-\frac{1}{s_{1} s_{4}}\left(\frac{s_{3} s_{6}}{t_{3}}+\frac{s_{2} s_{5}}{t_{1}}-t_{2}\right) \\
& +\frac{2}{s_{1}}\left(1-\frac{s_{6}}{t_{3}}-\frac{s_{2}}{t_{1}}\right)+\frac{2}{s_{2}}\left(1-\frac{s_{1}}{t_{1}}-\frac{s_{3}}{t_{2}}\right)+\frac{2}{s_{3}}\left(1-\frac{s_{2}}{t_{2}}-\frac{s_{4}}{t_{3}}\right) \\
& +\frac{2}{s_{4}}\left(1-\frac{s_{3}}{t_{3}}-\frac{s_{5}}{t_{1}}\right)+\frac{2}{s_{5}}\left(1-\frac{s_{4}}{t_{1}}-\frac{s_{6}}{t_{2}}\right)+\frac{2}{s_{6}}\left(1-\frac{s_{5}}{t_{2}}-\frac{s_{1}}{t_{3}}\right) \\
& -4\left(\frac{1}{t_{1}}+\frac{1}{t_{2}}+\frac{1}{t_{3}}\right)+\cdots,
\end{aligned}
$$

where dots represent terms suppressed by a factor of order $\mathcal{O}\left(\zeta(2) \alpha^{\prime 2}\right.$ ) with respect to the leading (QCD) contribution. Actually, up to the last term, which is invariant under cyclic permutations, the leading contribution of (A.14) is the cyclicized version of the expansion of $L_{5}^{Y}$ given in (A.4). Indeed $L_{5}^{Z}$ is invariant under cyclic permutations to all orders in $\alpha^{\prime}$.

The functions $G_{i}$ entering (3.17) are:

$$
\begin{aligned}
G_{1}^{Z} & =\int_{0}^{1} d x \int_{0}^{1} d y \int_{0}^{1} d z\left(\frac{1-s_{14}}{x}-\frac{s_{14}}{1-x}\right) \frac{x y \mathcal{I}(x, y, z)}{(1-x y)(1-x y z)}, \\
G_{2}^{Z} & =\int_{0}^{1} d x \int_{0}^{1} d y \int_{0}^{1} d z\left(\frac{1-s_{34}}{1-x}-\frac{s_{34}}{x}\right) \frac{\mathcal{I}(x, y, z)}{y z(1-x)}, \\
G_{3}^{Z} & =s_{24} \int_{0}^{1} d x \int_{0}^{1} d y \int_{0}^{1} d z \frac{\mathcal{I}(x, y, z)}{x y z(1-x)(1-x y)(1-x y z)},
\end{aligned}
$$




$$
\begin{aligned}
G_{4}^{Z} & =\int_{0}^{1} d x \int_{0}^{1} d y \int_{0}^{1} d z\left(1-\frac{s_{12}}{x}\right) \frac{y \mathcal{I}(x, y, z)}{(1-y)(1-x y)(1-y z)(1-x y z)}, \\
G_{5}^{Z} & =s_{13} \int_{0}^{1} d x \int_{0}^{1} d y \int_{0}^{1} d z \frac{y \mathcal{I}(x, y, z)}{x(1-x)(1-y)(1-y z)}, \\
G_{6}^{Z} & =\int_{0}^{1} d x \int_{0}^{1} d y \int_{0}^{1} d z\left(\frac{1-s_{23}}{x}-\frac{s_{23}}{1-x}\right) \frac{\mathcal{I}(x, y, z)}{x y z(1-y)(1-y z)} .
\end{aligned}
$$

Their low-energy expansions are

$$
\begin{aligned}
G_{1}^{Z}= & -\frac{s_{2}+s_{5}-t_{1}-t_{2}}{s_{1} s_{3} t_{3}}+\cdots, \\
G_{2}^{Z}= & \frac{1}{s_{3} s_{6}}-\frac{1}{s_{6} t_{3}}-\frac{2}{s_{6} t_{2}}-\frac{s_{2}}{s_{3} s_{6} t_{2}}-\frac{s_{3}}{s_{2} s_{6} t_{2}}-\frac{s_{4}}{s_{3} s_{6} t_{3}}+\cdots, \\
G_{3}^{Z}= & s_{24}\left(\frac{1}{s_{2} s_{6} t_{2}}+\frac{1}{s_{3} s_{6} t_{2}}+\frac{1}{s_{1} s_{3} t_{3}}+\frac{1}{s_{3} s_{6} t_{3}}\right)+\cdots, \\
G_{4}^{Z}= & \frac{1}{s_{1} s_{4}}-\frac{1}{s_{4} t_{3}}-\frac{2}{s_{4} t_{1}}-\frac{s_{1}}{s_{2} s_{4} t_{1}}-\frac{s_{2}}{s_{1} s_{4} t_{1}}-\frac{s_{6}}{s_{1} s_{4} t_{3}}+\cdots, \\
G_{5}^{Z}= & s_{13}\left(\frac{1}{s_{1} s_{4} t_{1}}+\frac{1}{s_{2} s_{4} t_{1}}+\frac{1}{s_{1} s_{3} t_{3}}+\frac{1}{s_{1} s_{4} t_{3}}\right)+\cdots, \\
G_{6}^{Z}= & \frac{1}{s_{2} s_{4}}+\frac{1}{s_{2} s_{6}}-\frac{2}{s_{4} s_{6}}-\frac{2}{s_{4} t_{1}}-\frac{2}{s_{6} t_{2}}-\frac{s_{1}}{s_{2} s_{4} t_{1}}-\frac{s_{2}}{s_{1} s_{4} t_{1}}-\frac{s_{2}}{s_{3} s_{6} t_{2}}-\frac{s_{3}}{s_{2} s_{6} t_{2}} \\
& -\frac{s_{2}}{s_{1} s_{3} t_{3}}-\frac{s_{2}}{s_{1} s_{4} t_{3}}-\frac{s_{2}}{s_{3} s_{6} t_{3}}-\frac{s_{2}}{s_{4} s_{6} t_{3}}-\frac{t_{3}}{s_{2} s_{4} t_{6}}+\cdots,
\end{aligned}
$$

where dots represent terms suppressed by a factor of $\operatorname{order} \mathcal{O}\left(\zeta(2) \alpha^{\prime 2}\right)$ with respect to the leading (QCD) contribution.

\section{Appendix B. Basis representation of the functions $N_{i}$}

In Ref. [3] it was shown that the full six-gluon amplitude can be expressed in a basis of six multiple hypergeometric functions. In $[4,5]$ we introduced a specific basis, $\left\{F_{1}, \ldots, F_{6}\right\}$ to describe the MHV amplitude. These six hypergeometric functions are represented by generalized Euler integrals:

$$
\begin{aligned}
& F_{1}=\int_{0}^{1} d x \int_{0}^{1} d y \int_{0}^{1} d z \frac{\mathcal{I}(x, y, z)}{x y z}, \quad F_{2}=\int_{0}^{1} d x \int_{0}^{1} d y \int_{0}^{1} d z \frac{\mathcal{I}(x, y, z)}{z(1-x y)}, \\
& F_{3}=\int_{0}^{1} d x \int_{0}^{1} d y \int_{0}^{1} d z \frac{\mathcal{I}(x, y, z)}{1-x y z}, \quad F_{4}=\int_{0}^{1} d x \int_{0}^{1} d y \int_{0}^{1} d z \frac{y \mathcal{I}(x, y, z)}{(1-x y)(1-y z)}, \\
& F_{5}=\int_{0}^{1} d x \int_{0}^{1} d y \int_{0}^{1} d z \frac{\mathcal{I}(x, y, z)}{(1-x y)(1-x y z)},
\end{aligned}
$$




$$
F_{6}=\int_{0}^{1} d x \int_{0}^{1} d y \int_{0}^{1} d z \frac{\mathcal{I}(x, y, z)}{(1-y z)(1-x y z)}
$$

In order to make contact between NMHV and MHV amplitudes, it would be desirable to express the NMHV functions, in particular the functions $N_{i}$ that enter the final result, in terms of this basis. Indeed, some NMHV functions are related to this basis in a simple way. For example:

$$
\begin{aligned}
& G_{1}^{Y}=s_{6} F_{2}+\left(s_{1}-s_{5}-s_{6}+t_{2}\right) F_{3}-s_{1} F_{5}, \quad G_{2}^{Y}=-s_{3} F_{1}, \\
& G_{3}^{Y}=-\left(s_{3}-t_{2}\right) F_{1}+\left(s_{6}-t_{3}\right) F_{2}-\left(s_{3}+s_{6}-t_{2}-t_{3}\right) F_{3}-s_{1} F_{5},
\end{aligned}
$$

however other relations are more complicated. Here, we focus on the functions $N_{i}$, see Eqs. (4.4), (4.8) and (4.12), which determine the NMHV amplitudes (4.3), (4.7) and (4.11), respectively.

\section{B.1. Helicity configuration $Y$}

$$
\begin{aligned}
N_{1}^{Y}= & s_{1} s_{3}\left(s_{4} t_{3}\right)^{-1}\left\{s_{2} s_{5} t_{2} F_{1}+s_{2} s_{5}\left(s_{4}+s_{5}-t_{1}\right) N_{0}-\left[s_{1} s_{5}+\left(s_{4}-t_{1}\right)\left(s_{5}+t_{3}\right)\right] \tilde{N}_{0}\right. \\
& -s_{5}\left[s_{3} s_{4}-s_{2}\left(s_{3}-t_{3}\right)\right] F_{2}-s_{5} s_{1}\left(s_{2}-t_{1}\right) F_{3}-s_{1} s_{4} s_{5} F_{5}-s_{3} s_{4} t_{3}\left(F_{3}-F_{5}\right) \\
& \left.+\left(s_{5}+t_{3}\right)\left[s_{4} t_{2} F_{2}+s_{2} s_{5} F_{3}+t_{1}\left(s_{4}-t_{1}\right) F_{3}+s_{4}\left(s_{4}-t_{1}\right)\left(F_{4}-F_{5}\right)+s_{4} s_{5} F_{4}\right]\right\}, \\
N_{2}^{Y}= & s_{1}\left(s_{4} t_{1}\right)^{-1}\left\{s_{6} t_{2} F_{1}+\left[s_{6}\left(s_{5}-t_{1}\right)-s_{4}\left(s_{5}-t_{2}\right)\right] N_{0}-\left(s_{1}-s_{3}\right) \tilde{N}_{0}\right. \\
& +\left[s_{5} s_{6}+t_{1}\left(s_{1}-t_{3}\right)\right] F_{3}-\left(s_{1}-s_{3}\right)\left(s_{4}+s_{6}\right) F_{3}-s_{4} s_{5}\left(F_{3}+F_{4}-F_{5}\right) \\
& \left.-\left(s_{3}-t_{3}\right)\left[t_{2} F_{3}+\left(s_{1}-s_{3}\right)\left(F_{3}-F_{5}\right)+\left(s_{5}-t_{1}\right)\left(F_{4}-F_{5}\right)\right]\right\}, \\
N_{3}^{Y}= & s_{3} F_{1}, \\
N_{4}^{Y}= & s_{1} s_{4}^{-1}\left\{t_{2}^{2} F_{1}+t_{2}\left(s_{4}+s_{5}-t_{1}\right) N_{0}-\left(s_{1}-s_{3}-s_{5}-t_{3}\right) \tilde{N}_{0}+s_{3} t_{2}\left(F_{2}-F_{3}\right)\right. \\
& -t_{2} t_{3} F_{2}+2 s_{3} s_{4} F_{3}+\left(s_{1}-s_{3}-s_{5}-t_{3}\right)\left(t_{1}-t_{2}\right) F_{3} \\
& \left.+s_{4}\left(s_{1}+s_{3}-s_{5}-t_{3}\right)\left(F_{4}-F_{5}\right)\right\}, \\
N_{5}^{Y}= & s_{3} s_{4}^{-1}\left\{t_{1} t_{2} F_{1}-\left(s_{3} s_{4}-s_{4} s_{5}-s_{4} t_{3}+t_{1} t_{3}\right)\left(F_{2}-F_{3}\right)+\left(s_{1} s_{4}+s_{4} s_{5}+s_{3} t_{1}\right) F_{2}\right. \\
& \left.+t_{1}\left(s_{4}+s_{5}-t_{1}\right) N_{0}+\left(s_{1} s_{4}-s_{1} t_{1}+s_{5} t_{1}\right) F_{3}\right\}, \\
N_{6}^{Y}= & s_{4}^{-1}\left\{t_{2} t_{3} F_{1}+\left[s_{4}\left(s_{1}+s_{3}-s_{5}\right)+t_{3}\left(s_{5}-t_{1}\right)\right] N_{0}+s_{3} t_{3} F_{2}-t_{3}^{2}\left(F_{2}-F_{3}\right)\right. \\
& \left.-\left(s_{1}-s_{5}\right) t_{3} F_{3}\right\},
\end{aligned}
$$

with the definitions:

$$
\begin{aligned}
N_{0}= & \frac{s_{6}}{s_{2}}\left(F_{2}-F_{3}\right)-\frac{s_{3}-s_{5}+t_{1}-t_{3}}{s_{2}}\left(F_{3}+F_{4}\right) \\
& -\frac{s_{1}-s_{3}+s_{5}-t_{1}}{s_{2}} F_{5}+\frac{s_{1}+s_{3}-s_{5}-t_{3}}{s_{2}} F_{6}, \\
\tilde{N}_{0}= & s_{6}\left(F_{2}-F_{3}\right)+\left(s_{1}-s_{3}+t_{2}\right) F_{3}+\left(s_{4}+s_{5}-t_{1}\right)\left(F_{4}-F_{5}\right)-\left(s_{1}-s_{3}\right) F_{5} .
\end{aligned}
$$

\section{B.2. Helicity configuration $X$}

$$
N_{1}^{X}=-s_{1} s_{3}\left\{s_{6}\left(F_{2}+F_{3}\right)+s_{1}\left(F_{3}-F_{5}\right)+\left(s_{5}-t_{1}\right)\left(F_{3}+F_{4}-F_{5}\right)-s_{5} F_{6}\right\},
$$




$$
\begin{aligned}
N_{2}^{X}= & s_{1}\left(s_{4} t_{1}\right)^{-1}\left\{s_{6} t_{2} F_{1}+\left[s_{6}\left(s_{5}-t_{1}\right)-s_{4}\left(s_{5}-t_{2}\right)\right] N_{0}-\left(s_{1}-s_{3}\right) \tilde{N}_{0}\right. \\
& -\left(s_{4}+s_{6}\right)\left(s_{1}-s_{3}\right) F_{3}-\left(s_{3}-t_{3}\right)\left(s_{5}-t_{1}\right)\left(F_{4}-F_{5}\right)+\left(s_{1}-s_{3}\right)\left(s_{3}-t_{3}\right)\left(F_{3}-F_{5}\right) \\
& \left.-s_{4} s_{5}\left(F_{3}+F_{4}-F_{5}\right)+\left[s_{5} s_{6}+t_{1}\left(s_{1}-t_{3}\right)-t_{2}\left(s_{3}-t_{3}\right)\right] F_{3}\right\}, \\
N_{3}^{X}= & s_{3} F_{1}, \\
N_{4}^{X}= & s_{1} s_{4}^{-1}\left\{s_{4} t_{2} N_{0}+t_{3} \tilde{N}_{0}-t_{1} t_{3} F_{3}-s_{4} t_{3}\left(F_{4}-F_{5}\right)+2 s_{3} s_{4}\left(F_{3}+F_{4}-F_{5}\right)\right\}, \\
N_{5}^{X}= & s_{3}\left\{t_{1} N_{0}+t_{3}\left(F_{2}-F_{3}\right)+2 s_{1} F_{3}\right\}, \\
N_{6}^{X}= & s_{4}^{-1}\left\{t_{2} t_{3} F_{1}+\left[s_{4}\left(s_{1}+s_{3}-s_{5}\right)+t_{3}\left(s_{5}-t_{1}\right)\right] N_{0}+s_{3} t_{3} F_{2}-t_{3}^{2}\left(F_{2}-F_{3}\right)\right. \\
& \left.-\left(s_{1}-s_{5}\right) t_{3} F_{3}\right\} .
\end{aligned}
$$

\section{B.3. Helicity configuration $Z$}

$$
\begin{aligned}
& s_{1} s_{3} s_{6}\left(s_{1}+s_{2}-t_{1}\right)^{-1}\left(s_{2}+s_{3}-t_{2}\right)^{-1} N_{1}^{Z} \\
& =\left[s_{6}\left(s_{1} s_{3} s_{5}+s_{2} s_{4} s_{6}+s_{3} s_{6} t_{1}+s_{1} s_{4} t_{2}\right)\right] \tilde{F}_{1} \\
& +\left\{-s_{2} s_{4} s_{5} s_{6}+\left[-s_{3} t_{1}^{2}+s_{3}\left(s_{1}+s_{2}+s_{4}\right) t_{1}+s_{4}\left(s_{2}^{2}+s_{1}\left(t_{2}-s_{3}\right)\right)\right] s_{6}+s_{1} s_{4}\right. \\
& \left.\times\left(s_{2}+s_{3}-t_{2}\right) t_{2}\right\} \tilde{F}_{2}+\left\{-s_{2} s_{6} s_{5}^{2}-s_{5} s_{6}\left[s_{2}\left(s_{2}+s_{3}+s_{6}-t_{1}-2 t_{2}\right)-s_{1} s_{3}\right]\right. \\
& +\left(s_{2}+s_{3}\right) s_{6}^{2} t_{1}-s_{1} t_{2}\left(s_{2}+s_{3}-t_{2}\right)\left(s_{1}-s_{3}+s_{4}-t_{1}+t_{2}\right)-s_{6}\left[\left(s_{4}-t_{1}\right) s_{2}^{2}\right. \\
& -\left(t_{1}\left(s_{3}-2 t_{2}\right)+s_{4} t_{2}\right) s_{2}+s_{3} t_{1}\left(-s_{3}+s_{4}-t_{1}+t_{2}\right)+s_{1}\left(s_{2}^{2}+2\left(s_{3}-t_{2}\right) s_{2}+s_{3}^{2}\right. \\
& \left.\left.\left.+t_{2}^{2}+s_{3}\left(-s_{4}+t_{1}-2 t_{2}\right)\right)\right]\right\} \tilde{F}_{3}+\left\{-\left(s_{2}+s_{3}-t_{2}\right)\left[s_{2} s_{5} s_{6}+\left(s_{1}\left(s_{2}+s_{3}-t_{2}\right)\right.\right.\right. \\
& \left.\left.\left.-\left(s_{2}+s_{3}\right) t_{1}\right) s_{6}+s_{1} t_{2}\left(s_{1}-s_{3}-t_{1}+t_{2}\right)\right]\right\} \tilde{F}_{4} \\
& +\left\{s_{2} s_{6} s_{5}^{2}+\left[-\left(s_{2}+s_{3}\right) s_{6} t_{1}+\left(s_{1}+s_{2}\right) s_{6}\left(s_{3}-t_{2}\right)+s_{1} t_{2}\left(-s_{2}-s_{3}+t_{2}\right)\right] s_{5}+s_{6} t_{1}\right. \\
& \times\left(\left(s_{1}+s_{2}+s_{3}\right) t_{2}-s_{3}\left(s_{2}+s_{3}\right)\right)+s_{1}\left(s_{2}+s_{3}-t_{2}\right) \\
& \left.\times\left(s_{6}\left(s_{2}+s_{3}-t_{2}\right)+t_{2}\left(s_{1}-s_{3}+t_{2}\right)\right)\right\} \tilde{F}_{5} \\
& +\left\{\left(s_{2}+s_{3}-t_{2}\right)\left[\left(s_{2}+s_{3}\right) s_{6}\left(s_{1}-t_{1}\right)+s_{1}\left(s_{1}-s_{3}-t_{1}\right) t_{2}+s_{5}\left(s_{2} s_{6}+s_{1} t_{2}\right)\right]\right\} \tilde{F}_{6}, \\
& s_{1} s_{2} s_{3} t_{1}\left(s_{1}+s_{2}-t_{1}\right)^{-1} N_{2}^{Z} \\
& =\left\{s_{2}\left(s_{3}+s_{4}\right) s_{6} t_{1}+\left(s_{1}+s_{2}\right) s_{3} s_{6} t_{3}\right\} \tilde{F}_{1} \\
& +\left\{-s_{2} s_{3} t_{1}^{2}+\left(s_{1}+s_{2}\right) s_{3}\left(-s_{1} s_{2}+s_{3} s_{4}-s_{4} s_{5}+s_{1} s_{6}+s_{2} t_{3}\right)\right. \\
& \left.+t_{1}\left(-s_{1} s_{3} s_{6}+s_{2}\left(2 s_{1} s_{3}+2 s_{3} s_{4}+s_{2}\left(s_{3}+s_{4}\right)-s_{4} s_{5}-s_{3} t_{3}\right)\right)\right\} \tilde{F}_{2} \\
& +\left\{s_{2} t_{1}^{2}\left(s_{2}+2 s_{3}+s_{5}+s_{6}-2 t_{2}\right)+s_{3}\left[-\left(-s_{1}+s_{2}+s_{3}-s_{4}+s_{5}+s_{6}-2 t_{2}\right)\right.\right. \\
& \left.\times\left(-s_{3}+s_{5}\right)-\left(s_{2}+s_{5}-t_{2}\right) t_{3}\right]\left(s_{1}+s_{2}\right)+t_{1}\left[-s_{2} s_{5}^{2}-s_{2} s_{5}\left(s_{2}+s_{6}-2 t_{2}\right)\right. \\
& +s_{1}\left(-s_{2}^{2}+s_{3}\left(s_{3}+s_{6}-t_{2}\right)+s_{2}\left(-2 s_{3}+t_{2}\right)\right)+s_{2}\left(2 s_{3}^{2}+s_{2}\left(s_{3}-s_{4}\right)\right. \\
& \left.\left.\left.+2 s_{3} s_{6}+s_{4} t_{2}+s_{3}\left(-2 s_{4}-3 t_{2}+t_{3}\right)\right)\right]\right\} \tilde{F}_{3} \\
& +\left\{\left(-s_{1}+s_{3}-s_{5}+t_{1}\right)\left[s_{1} s_{3}+s_{2}\left(s_{3}+t_{1}\right)\right]\left(s_{2}+s_{3}-t_{2}\right)\right\} \tilde{F}_{4} \\
& +\left\{-s_{2} t_{1}^{2}\left(s_{3}+s_{5}-t_{2}\right)+\left(s_{1}+s_{2}\right) s_{3}\left(-s_{3}+s_{5}\right)\left(-s_{1}+s_{3}+s_{5}-t_{2}\right)\right. \\
& \left.+s_{2} t_{1}\left[s_{5}^{2}+s_{1}\left(s_{2}+3 s_{3}-t_{2}\right)+2 s_{3}\left(-s_{3}+t_{2}\right)-s_{5}\left(s_{3}+t_{2}\right)\right]\right\} \tilde{F}_{5}
\end{aligned}
$$




$$
\begin{aligned}
& +\left\{\left(s_{1}-s_{3}+s_{5}-t_{1}\right)\left[s_{1} s_{3}+s_{2}\left(s_{3}+t_{1}\right)\right]\left(s_{2}+s_{3}-t_{2}\right)\right\} \tilde{F}_{6}, \\
& s_{1} s_{3} t_{3}\left(s_{2}+s_{3}-t_{2}\right)^{-1} N_{3}^{Z} \\
& =\left\{s_{2}\left(s_{1}+s_{6}\right) t_{2}+s_{1}\left(s_{2}+s_{3}\right) t_{3}\right\} F_{1} \\
& +\left\{-\left(s_{1}+s_{6}\right)\left(-s_{2} s_{3}+s_{3} s_{4}+s_{1} s_{6}-s_{5} s_{6}-s_{4} t_{2}\right)\right. \\
& \left.+\left[-s_{5} s_{6}+s_{1}\left(2 s_{3}+s_{4}+2 s_{6}-t_{2}\right)\right] t_{3}-s_{1} t_{3}^{2}\right\} F_{2} \\
& +\left\{\left(s_{1}+s_{6}\right)\left[-\left(s_{1}-s_{5}\right)\left(s_{1}+s_{2}-s_{3}+s_{4}+s_{5}-s_{6}-2 t_{1}\right)-\left(s_{1}+s_{4}-t_{1}\right) t_{2}\right]\right. \\
& +\left[s_{1}^{2}-s_{5}\left(s_{4}+s_{5}-s_{6}-2 t_{1}\right)+s_{3} t_{1}-s_{2}\left(s_{3}+s_{5}-t_{2}\right)+\left(s_{4}+s_{5}-2 t_{1}\right) t_{2}\right. \\
& \left.\left.+s_{1}\left(2 s_{2}-2 s_{3}+s_{4}+s_{5}-2 s_{6}-3 t_{1}+2 t_{2}\right)\right] t_{3}+s_{1} t_{3}^{2}\right\} F_{3} \\
& +\left\{-\left(s_{4}+s_{5}-t_{1}\right)\left[\left(s_{1}+s_{3}-s_{5}\right)\left(s_{1}+s_{6}\right)+\left(-2 s_{1}+s_{5}-t_{2}\right) t_{3}\right]\right\} F_{4} \\
& +\left\{\left(s_{1}-s_{5}\right)\left(s_{1}+s_{6}\right)\left(s_{1}-s_{3}+s_{5}-t_{1}\right)-\left[2 s_{1}^{2}-s_{2} s_{3}+s_{3} t_{1}\right.\right. \\
& \left.\left.-\left(s_{5}-t_{1}\right)\left(s_{5}-t_{2}\right)+s_{1}\left(-3 s_{3}+s_{5}-2 t_{1}+t_{2}\right)\right] t_{3}\right\} F_{5} \\
& +\left(s_{4}+s_{5}-t_{1}\right)\left[\left(s_{1}+s_{3}-s_{5}\right)\left(s_{1}+s_{6}\right)+\left(-2 s_{1}+s_{5}-t_{2}\right) t_{3}\right] F_{6} \text {, } \\
& s_{1} s_{2} s_{3} s_{6}\left(s_{1}+s_{2}-t_{1}\right)^{-1} N_{4}^{Z} \\
& =\left\{-s_{2} s_{3} s_{5} s_{6}-s_{2} s_{3} s_{6}^{2}+s_{1} t_{2}\left[s_{2}\left(s_{3}+s_{4}\right)+s_{3} t_{3}\right]\right. \\
& \left.+s_{2} s_{6}\left[-s_{1} s_{3}+s_{3}^{2}+s_{2}\left(s_{3}+2 s_{4}\right)-s_{4} t_{2}+s_{3}\left(s_{4}+t_{1}+t_{3}\right)\right]\right\} s_{6} \tilde{F}_{1} \\
& +\left\{-s_{2} s_{3} s_{6}^{2}\left(s_{1}+s_{2}-t_{1}\right)+s_{1}\left(s_{2}+s_{3}\right) s_{4}\left(s_{2}+s_{3}-t_{2}\right) t_{2}\right. \\
& -s_{2} s_{5} s_{6}\left[s_{1} s_{3}+2 s_{3} s_{4}+s_{2}\left(s_{3}+2 s_{4}\right)-s_{3} t_{1}-s_{4} t_{2}\right]+s_{6}\left[s_{1}^{2} s_{3}\left(-s_{2}+t_{2}\right)\right. \\
& +s_{2}\left[s_{2}^{2}\left(s_{3}+2 s_{4}\right)+s_{2}\left(s_{3}^{2}-s_{4} t_{2}+s_{3}\left(3 s_{4}+t_{3}\right)\right)+s_{3}\left(s_{3}\left(2 s_{4}-t_{1}\right)+s_{4}\left(t_{1}-t_{2}\right)\right.\right. \\
& \left.\left.\left.\left.-t_{1}\left(t_{1}+t_{3}\right)\right)\right]+s_{1}\left[s_{3}\left(s_{4}-t_{1}\right) t_{2}+s_{2}\left(s_{3}^{2}+s_{4} t_{2}+s_{3}\left(-s_{4}+2 t_{1}+t_{2}+t_{3}\right)\right)\right]\right]\right\} \tilde{F}_{2} \\
& +\left\{s_{2} s_{6}^{2}\left[s_{1} s_{3}+s_{2}\left(3 s_{3}+2 t_{1}\right)+\left(2 s_{3}+t_{1}\right)\left(s_{3}-t_{2}\right)\right]+s_{2} s_{5}^{2} s_{6}\left(-2 s_{2}-s_{3}+t_{2}\right)\right. \\
& -s_{1}\left(s_{2}+s_{3}\right)\left(s_{2}+s_{3}-t_{2}\right) t_{2}\left(s_{1}-s_{3}+s_{4}-t_{1}+t_{2}\right)-s_{2} s_{5} s_{6}\left[2 s_{2}^{2}-2 s_{1} s_{3}+s_{3}^{2}\right. \\
& \left.+s_{2}\left(2 s_{3}-2 t_{1}-5 t_{2}\right)+s_{6}\left(2 s_{2}+s_{3}-t_{2}\right)+t_{2}\left(t_{1}+2 t_{2}\right)+s_{3}\left(-s_{4}-3 t_{2}+t_{3}\right)\right] \\
& +s_{6}\left[s _ { 1 } \left[s_{1} s_{3}\left(s_{2}-t_{2}\right)-2 s_{2}^{3}+4 s_{2}^{2}\left(-s_{3}+t_{2}\right)+s_{3} t_{2}\left(s_{3}-s_{4}+t_{1}-t_{2}+t_{3}\right)\right.\right. \\
& \left.-s_{2}\left[3 s_{3}^{2}+2 t_{2}^{2}+s_{3}\left(-s_{4}+2 t_{1}-4 t_{2}+t_{3}\right)\right]\right]+s_{2}\left[2 s_{3}^{3}+s_{2}^{2}\left(s_{3}-2 s_{4}+2 t_{1}\right)\right. \\
& +s_{3}^{2}\left(-2 s_{4}+3 t_{1}-4 t_{2}\right)-\left(s_{4}-2 t_{1}\right) t_{2}^{2}+s_{2}\left[3 s_{3}^{2}+\left(3 s_{4}-5 t_{1}\right) t_{2}\right. \\
& \left.\left.\left.\left.-s_{3}\left(3 s_{4}-4 t_{1}+4 t_{2}+t_{3}\right)\right]+s_{3}\left[t_{1}^{2}+t_{1}\left(-s_{4}-4 t_{2}+t_{3}\right)+t_{2}\left(2\left(s_{4}+t_{2}\right)+t_{3}\right)\right]\right]\right]\right\} \tilde{F}_{3} \\
& +\left\{( s _ { 2 } + s _ { 3 } - t _ { 2 } ) \left[s_{1}\left(s_{2}+s_{3}\right)\left(-s_{1}+s_{3}+t_{1}-t_{2}\right) t_{2}+s_{2} s_{5} s_{6}\left(-2\left(s_{2}+s_{3}\right)+t_{2}\right)\right.\right. \\
& \left.\left.+s_{6}\left[-2 s_{1} s_{2}\left(s_{2}+s_{3}\right)+s_{2}\left(s_{3}+t_{1}\right)\left(2\left(s_{2}+s_{3}\right)-t_{2}\right)+s_{1}\left(2 s_{2}+s_{3}\right) t_{2}\right]\right]\right\} \tilde{F}_{4} \\
& +\left\{s_{2} s_{5}^{2} s_{6}\left(2\left(s_{2}+s_{3}\right)-t_{2}\right)+s_{1}\left(s_{2}+s_{3}\right)\left(s_{2}+s_{3}-t_{2}\right) t_{2}\left(s_{1}-s_{3}+t_{2}\right)\right. \\
& -s_{5}\left[s_{1}\left(s_{2}+s_{3}\right)\left(s_{2}+s_{3}-t_{2}\right) t_{2}+s_{6}\left(s_{1}\left(s_{2}+s_{3}\right) t_{2}+s_{2}\left[2\left(s_{2}+s_{3}\right)-t_{2}\right]\left(t_{1}+t_{2}\right)\right)\right] \\
& +s_{6}\left[-s_{2}\left(s_{3}+t_{1}\right)\left(s_{3}-t_{2}\right)\left(2\left(s_{2}+s_{3}\right)-t_{2}\right)+s_{1}^{2} s_{3} t_{2}+s_{1}\left[2 s_{2}^{3}+s_{2}^{2}\left(6 s_{3}-4 t_{2}\right)\right.\right. \\
& \left.\left.\left.+s_{3} t_{2}\left(-s_{3}+t_{2}\right)+s_{2}\left[4 s_{3}^{2}-5 s_{3} t_{2}+t_{2}\left(t_{1}+2 t_{2}\right)\right]\right]\right]\right\} \tilde{F}_{5} \\
& +\left\{\left(s_{1}-s_{3}+s_{5}-t_{1}\right)\left(s_{2}+s_{3}-t_{2}\right)\left[s_{2} s_{6}\left(2\left(s_{2}+s_{3}\right)-t_{2}\right)+s_{1}\left(s_{2}+s_{3}\right) t_{2}\right]\right\} \tilde{F}_{6} \text {, } \\
& s_{1} s_{2} s_{3} s_{6}\left(s_{2}+s_{3}-t_{2}\right)^{-1} N_{5}^{Z}
\end{aligned}
$$




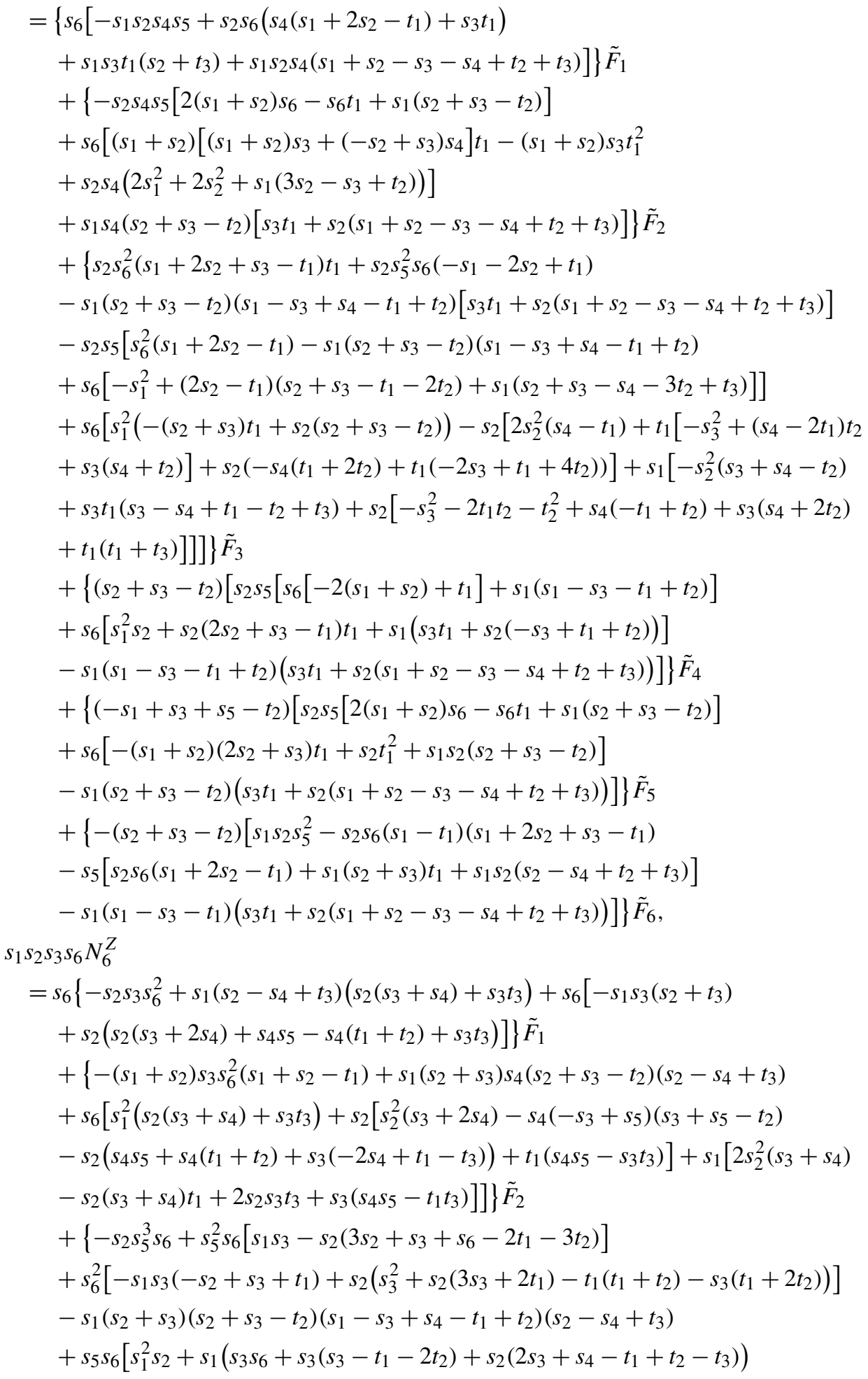




$$
\begin{aligned}
& +s_{2}\left[-2 s_{2}^{2}+s_{3}^{2}-s_{2}\left(s_{3}+s_{4}-4 t_{1}-5 t_{2}\right)+\left(s_{4}-2 t_{2}\right) t_{2}+s_{6}\left(-2 s_{2}+s_{3}+2 t_{1}+t_{2}\right)\right. \\
& \left.\left.-t_{1}\left(t_{1}+5 t_{2}\right)-s_{3}\left(-2 t_{1}+t_{3}\right)\right]\right]-s_{6}\left[s_{1}^{3} s_{3}\right. \\
& +s_{1}^{2}\left[-s_{2}^{2}+s_{2}\left(t_{1}+t_{2}\right)+s_{3}\left(s_{4}-t_{1}+t_{2}-t_{3}\right)\right] \\
& +s_{1}\left[s_{3}^{3}+s_{2}\left(s_{2}\left(t_{1}-t_{2}\right)+t_{2}^{2}+t_{1}\left(s_{4}-t_{1}-t_{3}\right)\right)+s_{3}^{2}\left(s_{4}+t_{1}-t_{2}-2 t_{3}\right)\right. \\
& \left.+s_{3}\left(s_{2}\left(s_{4}+t_{1}+t_{2}-2 t_{3}\right)+t_{2}\left(-2 t_{1}+t_{3}\right)\right)\right]+s_{2}\left(-s_{3}^{3}-s_{2}^{2}\left(s_{3}-2 s_{4}+2 t_{1}\right)\right. \\
& +\left(s_{4}-2 t_{1}\right) t_{2}\left(t_{1}+t_{2}\right)+s_{3}^{2}\left(s_{4}+3 t_{2}\right)+s_{3}\left[t_{1}^{2}+t_{1}\left(t_{2}-t_{3}\right)-t_{2}\left(s_{4}+2 t_{2}+t_{3}\right)\right] \\
& \left.\left.\left.+s_{2}\left(-3 s_{3}^{2}-s_{4}\left(t_{1}+3 t_{2}\right)+t_{1}\left(t_{1}+5 t_{2}\right)+s_{3}\left(2 s_{4}-3 t_{1}+4 t_{2}+t_{3}\right)\right)\right)\right]\right\} \tilde{F}_{3} \\
& +\left\{( s _ { 2 } + s _ { 3 } - t _ { 2 } ) \left[-s_{2} s_{5}^{2} s_{6}+s_{5} s_{6}\left(s_{1}\left(-s_{2}+s_{3}\right)+s_{2}\left(-2 s_{2}+2 t_{1}+t_{2}\right)\right)\right.\right. \\
& +s_{6}\left[s_{1}^{2}\left(s_{2}+2 s_{3}\right)+s_{2}\left(s_{3}+t_{1}\right)\left(2 s_{2}+s_{3}-t_{1}-t_{2}\right)+s_{1}\left(-2 s_{3} t_{1}+s_{2}\left(2 s_{3}+t_{2}\right)\right)\right] \\
& \left.\left.+s_{1}\left(s_{2}+s_{3}\right)\left(-s_{1}+s_{3}+t_{1}-t_{2}\right)\left(s_{2}-s_{4}+t_{3}\right)\right]\right\} \tilde{F}_{4} \\
& +\left\{( - s _ { 1 } + s _ { 3 } + s _ { 5 } - t _ { 2 } ) \left[s_{2} s_{5}^{2} s_{6}+s_{5} s_{6}\left(s_{1}\left(s_{2}-s_{3}\right)+s_{2}\left(2 s_{2}-2 t_{1}-t_{2}\right)\right)\right.\right. \\
& -s_{6}\left(s_{1}^{2} s_{3}+s_{2}\left(s_{3}+t_{1}\right)\left(2 s_{2}+s_{3}-t_{1}-t_{2}\right)+s_{1}\left(-s_{2}^{2}-s_{3} t_{1}+s_{2}\left(s_{3}+t_{1}+t_{2}\right)\right)\right) \\
& \left.\left.-s_{1}\left(s_{2}+s_{3}\right)\left(s_{2}+s_{3}-t_{2}\right)\left(s_{2}-s_{4}+t_{3}\right)\right]\right\} \tilde{F}_{5} \\
& \times\left\{( s _ { 1 } - s _ { 3 } + s _ { 5 } - t _ { 1 } ) ( s _ { 2 } + s _ { 3 } - t _ { 2 } ) \left[s_{2} s_{5} s_{6}+s_{6}\left[-s_{1} s_{3}+s_{2}\left(2 s_{2}+s_{3}-t_{1}-t_{2}\right)\right]\right.\right. \\
& \left.\left.+s_{1}\left(s_{2}+s_{3}\right)\left(s_{2}-s_{4}+t_{3}\right)\right]\right\} \tilde{F}_{6},
\end{aligned}
$$

with

$$
\begin{aligned}
\tilde{F}_{3}= & F_{3}+F_{4}-F_{6}, \quad \tilde{F}_{4}=F_{6}, \quad \tilde{F}_{5}=F_{4}, \quad \tilde{F}_{6}=F_{5}, \\
\tilde{F}_{2}= & \frac{s_{6}}{s_{4}} F_{2}+\left(1+\frac{s_{1}}{s_{4}}-\frac{s_{6}}{s_{4}}-\frac{t_{1}}{s_{4}}+\frac{t_{2}}{s_{4}}-\frac{s_{3}}{s_{4}}\right) F_{3}+\left(1+\frac{s_{5}}{s_{4}}-\frac{t_{1}}{s_{4}}\right) F_{4} \\
& +\left(-\frac{s_{1}}{s_{4}}-\frac{s_{5}}{s_{4}}+\frac{t_{1}}{s_{4}}+\frac{s_{3}}{s_{4}}\right) F_{5}-F_{6}, \\
\tilde{F}_{1}= & \frac{s_{2} t_{2}}{s_{4} t_{3}} F_{1}+\left(-\frac{s_{2}}{s_{4}}+\frac{s_{3} s_{2}}{s_{4} t_{3}}-\frac{s_{3}}{t_{3}}+\frac{s_{5} s_{6}}{s_{4} t_{3}}-\frac{s_{1} s_{6}}{s_{4} t_{3}}+\frac{t_{2}}{t_{3}}\right) F_{2} \\
& +\left(-\frac{s_{1}^{2}}{s_{4} t_{3}}+\frac{s_{6} s_{1}}{s_{4} t_{3}}+\frac{2 t_{1} s_{1}}{s_{4} t_{3}}-\frac{t_{2} s_{1}}{s_{4} t_{3}}-\frac{s_{2} s_{1}}{s_{4} t_{3}}+\frac{s_{3} s_{1}}{s_{4} t_{3}}-\frac{s_{1}}{t_{3}}+\frac{s_{5}}{s_{4}}-\frac{t_{1}}{s_{4}}+\frac{s_{2}}{s_{4}}+\frac{s_{5}^{2}}{s_{4} t_{3}}\right. \\
& \left.+\frac{s_{2} s_{5}}{s_{4} t_{3}}-\frac{s_{3} s_{5}}{s_{4} t_{3}}+\frac{s_{5}}{t_{3}}-\frac{s_{5} s_{6}}{s_{4} t_{3}}-\frac{2 s_{5} t_{1}}{s_{4} t_{3}}+\frac{t_{1} t_{2}}{s_{4} t_{3}}-\frac{t_{2}}{t_{3}}\right) F_{3} \\
& +\left(\frac{s_{5}^{2}}{s_{4} t_{3}}+\frac{s_{5}}{s_{4}}-\frac{t_{1} s_{5}}{s_{4} t_{3}}-\frac{s_{1} s_{5}}{s_{4} t_{3}}-\frac{s_{3} s_{5}}{s_{4} t_{3}}+\frac{s_{5}}{t_{3}}-\frac{t_{1}}{s_{4}}-\frac{s_{1}}{t_{3}}-\frac{s_{3}}{t_{3}}+\frac{s_{1} t_{1}}{s_{4} t_{3}}+\frac{s_{3} t_{1}}{s_{4} t_{3}}\right) F_{4} \\
& +\left(\frac{s_{1}^{2}}{s_{4} t_{3}}-\frac{t_{1} s_{1}}{s_{4} t_{3}}-\frac{s_{3} s_{1}}{s_{4} t_{3}}-\frac{s_{5}^{2}}{s_{4} t_{3}}+\frac{s_{3} s_{5}}{s_{4} t_{3}}+\frac{s_{5} t_{1}}{s_{4} t_{3}}\right) F_{5} \\
& +\left(-\frac{s_{5}^{2}}{s_{4} t_{3}}-\frac{s_{5}}{s_{4}}+\frac{t_{1} s_{5}}{s_{4} t_{3}}+\frac{s_{1} s_{5}}{s_{4} t_{3}}+\frac{s_{3} s_{5}}{s_{4} t_{3}}-\frac{s_{5}}{t_{3}}+\frac{t_{1}}{s_{4}}+\frac{s_{1}}{t_{3}}+\frac{s_{3}}{t_{3}}-\frac{s_{1} t_{1}}{s_{4} t_{3}}-\frac{s_{3} t_{1}}{s_{4} t_{3}}\right) F_{6} .
\end{aligned}
$$


Finally, we list the next order of the expansions (4.13). It comes with a factor of $\zeta(2)$ :

$$
\begin{aligned}
& N_{1}^{Z}=\cdots+\zeta(2)\left(s_{1}+s_{2}-t_{1}\right)\left(s_{2}+s_{3}-t_{2}\right)\left\{-\frac{t_{1}^{2}}{s_{1} s_{4}}+\frac{t_{1}}{s_{1}}+\frac{t_{1}}{s_{4}}-\frac{s_{3} t_{1}}{s_{1} t_{3}}-\frac{s_{6} t_{1}}{s_{4} t_{3}}-\frac{t_{2}^{2}}{s_{3} s_{6}}\right. \\
& \left.-\frac{s_{2} s_{5}}{s_{1} s_{3}}+\frac{t_{2}}{s_{3}}+\frac{t_{2}}{s_{6}}-\frac{s_{2} s_{5}}{s_{4} s_{6}}-\frac{s_{2} s_{4}}{s_{1} t_{3}}-\frac{s_{1} s_{5}}{s_{4} t_{3}}-\frac{s_{2} s_{6}}{s_{3} t_{3}}-\frac{s_{1} t_{2}}{s_{3} t_{3}}-\frac{s_{4} t_{2}}{s_{6} t_{3}}-\frac{s_{3} s_{5}}{s_{6} t_{3}}-1\right\}+\cdots, \\
& N_{2}^{Z}=\cdots+\zeta(2)\left(s_{1}+s_{2}-t_{1}\right)\left\{-\frac{s_{1}}{s_{4} t_{1}}-\frac{s_{6}}{s_{2} s_{4}}+\frac{2}{s_{4}}-\frac{s_{5}}{s_{2} t_{1}}-\frac{s_{2}}{s_{4} t_{1}}-\frac{s_{6}}{s_{3} t_{3}}-\frac{s_{6}}{s_{4} t_{3}}-\frac{s_{5}}{s_{3} s_{1}}\right. \\
& \left.-\frac{t_{1}}{s_{4} s_{1}}-\frac{t_{3}}{s_{4} s_{1}}-\frac{s_{5}}{t_{1} s_{1}}-\frac{s_{3}}{t_{3} s_{1}}-\frac{s_{4}}{t_{3} s_{1}}+\frac{2}{s_{1}}\right\}+\cdots, \\
& N_{3}^{Z}=\cdots+\zeta(2)\left(s_{2}+s_{3}-t_{2}\right)\left\{-\frac{s_{1}}{s_{3} t_{3}}-\frac{t_{2}}{s_{3} s_{6}}-\frac{t_{3}}{s_{3} s_{6}}+\frac{2}{s_{3}}-\frac{s_{4}}{s_{2} s_{6}}+\frac{2}{s_{6}}-\frac{s_{5}}{s_{2} t_{2}}-\frac{s_{5}}{s_{3} t_{2}}\right. \\
& \left.-\frac{s_{2}}{s_{6} t_{2}}-\frac{s_{3}}{s_{6} t_{2}}-\frac{s_{6}}{s_{3} t_{3}}-\frac{s_{4}}{s_{6} t_{3}}-\frac{s_{5}}{s_{3} s_{1}}-\frac{s_{4}}{t_{3} s_{1}}\right\}+\cdots, \\
& N_{4}^{Z}=\cdots+\zeta(2)\left(s_{1}+s_{2}-t_{1}\right)\left\{-\frac{s_{3}^{2}}{s_{1} t_{3}}-\frac{t_{1} s_{3}}{s_{1} s_{4}}+\frac{s_{3}}{s_{1}}+\frac{s_{3}}{s_{4}}-\frac{s_{2} s_{3}}{s_{1} t_{3}}-\frac{s_{4} s_{3}}{s_{1} t_{3}}+\frac{s_{5} s_{3}}{s_{1} t_{3}}+\frac{s_{6} s_{3}}{s_{1} t_{3}}\right. \\
& -\frac{s_{6} s_{3}}{s_{4} t_{3}}-\frac{t_{1} s_{3}}{s_{1} t_{3}}-\frac{t_{2} s_{3}}{s_{6} t_{3}}+\frac{s_{3}}{t_{3}}-\frac{t_{1}^{2}}{s_{1} s_{4}}-\frac{t_{2}^{2}}{s_{2} s_{6}}+\frac{2 s_{2}}{s_{1}}-\frac{2 s_{5}}{s_{1}}-\frac{s_{5}}{s_{4}}-\frac{2 s_{6}}{s_{4}}+\frac{s_{5} t_{1}}{s_{1} s_{4}} \\
& +\frac{s_{6} t_{1}}{s_{1} s_{4}}+\frac{t_{1}}{s_{1}}-\frac{s_{2} t_{1}}{s_{1} s_{4}}+\frac{2 t_{1}}{s_{4}}-\frac{t_{1} t_{2}}{s_{2} s_{4}}-\frac{t_{2}}{s_{1}}+\frac{t_{2}}{s_{2}}+\frac{t_{2}}{s_{4}}-\frac{s_{2} t_{2}}{s_{4} s_{6}}+\frac{2 t_{2}}{s_{6}}-\frac{t_{1} t_{3}}{s_{1} s_{4}}-\frac{t_{2} t_{3}}{s_{4} s_{6}} \\
& +\frac{t_{3}}{s_{4}}-\frac{s_{1}}{s_{4}}+\frac{s_{2}}{s_{4}}+\frac{s_{6}^{2}}{s_{4} t_{3}}-\frac{2 s_{2} s_{4}}{s_{1} t_{3}}+\frac{s_{5} s_{6}}{s_{4} t_{3}}+\frac{s_{1} s_{6}}{s_{4} t_{3}}-\frac{s_{2} s_{6}}{s_{4} t_{3}}-\frac{s_{6}}{t_{3}}-\frac{s_{6} t_{1}}{s_{4} t_{3}}+\frac{s_{4} t_{2}}{s_{1} t_{3}} \\
& \left.-\frac{s_{1} t_{2}}{s_{4} t_{3}}-\frac{s_{4} t_{2}}{s_{6} t_{3}}-1-\frac{t_{2}^{2}}{s_{6} s_{3}}-\frac{2 s_{2} s_{5}}{s_{1} s_{3}}+\frac{s_{5} t_{2}}{s_{1} s_{3}}+\frac{t_{2}}{s_{3}}-\frac{2 s_{2} s_{6}}{t_{3} s_{3}}-\frac{s_{1} t_{2}}{t_{3} s_{3}}+\frac{s_{6} t_{2}}{t_{3} s_{3}}\right\}+\cdots, \\
& N_{5}^{Z}=\cdots+\zeta(2)\left(s_{2}+s_{3}-t_{2}\right)\left\{-\frac{s_{1}^{2}}{s_{3} t_{3}}-\frac{t_{2} s_{1}}{s_{3} s_{6}}+\frac{s_{1}}{s_{3}}+\frac{s_{1}}{s_{6}}+\frac{s_{4} s_{1}}{s_{3} t_{3}}+\frac{s_{5} s_{1}}{s_{3} t_{3}}-\frac{s_{6} s_{1}}{s_{3} t_{3}}-\frac{t_{1} s_{1}}{s_{4} t_{3}}\right. \\
& -\frac{t_{2} s_{1}}{s_{3} t_{3}}-\frac{s_{2} s_{1}}{s_{3} t_{3}}-\frac{s_{4} s_{1}}{s_{6} t_{3}}+\frac{s_{1}}{t_{3}}-\frac{t_{1}^{2}}{s_{2} s_{4}}-\frac{t_{2}^{2}}{s_{3} s_{6}}-\frac{2 s_{5}}{s_{3}}+\frac{t_{1}}{s_{2}}-\frac{t_{1}}{s_{3}}+\frac{2 t_{1}}{s_{4}}-\frac{s_{2} t_{1}}{s_{4} s_{6}}+\frac{t_{1}}{s_{6}} \\
& -\frac{t_{1} t_{2}}{s_{2} s_{6}}+\frac{t_{2}}{s_{3}}+\frac{s_{4} t_{2}}{s_{3} s_{6}}+\frac{s_{5} t_{2}}{s_{3} s_{6}}-\frac{s_{2} t_{2}}{s_{3} s_{6}}+\frac{2 t_{2}}{s_{6}}-\frac{t_{1} t_{3}}{s_{4} s_{6}}-\frac{t_{2} t_{3}}{s_{3} s_{6}}+\frac{t_{3}}{s_{6}}+\frac{2 s_{2}}{s_{3}}+\frac{s_{2}}{s_{6}}-\frac{s_{3}}{s_{6}} \\
& -\frac{2 s_{4}}{s_{6}}-\frac{s_{5}}{s_{6}}-\frac{s_{4}}{t_{3}}-\frac{2 s_{2} s_{6}}{s_{3} t_{3}}+\frac{s_{6} t_{1}}{s_{3} t_{3}}-\frac{s_{6} t_{1}}{s_{4} t_{3}}-\frac{s_{3} t_{1}}{s_{6} t_{3}}-\frac{s_{4} t_{2}}{s_{6} t_{3}}+\frac{s_{4}^{2}}{s_{6} t_{3}}-\frac{s_{2} s_{4}}{s_{6} t_{3}} \\
& \left.+\frac{s_{3} s_{4}}{s_{6} t_{3}}+\frac{s_{4} s_{5}}{s_{6} t_{3}}-1-\frac{t_{1}^{2}}{s_{4} s_{1}}-\frac{2 s_{2} s_{5}}{s_{3} s_{1}}+\frac{s_{5} t_{1}}{s_{3} s_{1}}+\frac{t_{1}}{s_{1}}-\frac{2 s_{2} s_{4}}{t_{3} s_{1}}-\frac{s_{3} t_{1}}{t_{3} s_{1}}+\frac{s_{4} t_{1}}{t_{3} s_{1}}\right\}+\cdots, \\
& N_{6}^{Z}=\cdots+\zeta(2)\left\{-\frac{s_{2}^{2}}{s_{4} s_{6}}-\frac{2 s_{5} s_{2}}{s_{1} s_{3}}-\frac{t_{1} s_{2}}{s_{1} s_{4}}-\frac{t_{2} s_{2}}{s_{3} s_{6}}-\frac{2 t_{3} s_{2}}{s_{4} s_{6}}+\frac{2 s_{2}}{s_{1}}+\frac{2 s_{2}}{s_{3}}+\frac{3 s_{2}}{s_{4}}+\frac{3 s_{2}}{s_{6}}\right. \\
& -\frac{s_{3} s_{2}}{s_{1} t_{3}}-\frac{2 s_{4} s_{2}}{s_{1} t_{3}}-\frac{2 s_{6} s_{2}}{s_{3} t_{3}}-\frac{s_{6} s_{2}}{s_{4} t_{3}}-\frac{s_{1} s_{2}}{s_{3} t_{3}}-\frac{s_{1} s_{2}}{s_{4} t_{3}}-\frac{s_{3} s_{2}}{s_{6} t_{3}}-\frac{s_{4} s_{2}}{s_{6} t_{3}}-\frac{s_{5}^{2}}{s_{1} s_{3}}-\frac{t_{3}^{2}}{s_{4} s_{6}} \\
& -\frac{3 s_{6}}{s_{4}}+\frac{s_{5} t_{1}}{s_{1} s_{3}}+\frac{s_{6} t_{1}}{s_{1} s_{4}}-\frac{t_{1}}{s_{3}}-\frac{t_{1}}{s_{4}}+\frac{s_{5} t_{2}}{s_{1} s_{3}}-\frac{t_{2}}{s_{1}}+\frac{s_{4} t_{2}}{s_{3} s_{6}}-\frac{t_{2}}{s_{6}}-\frac{t_{1} t_{3}}{s_{1} s_{4}}-\frac{t_{2} t_{3}}{s_{3} s_{6}}+\frac{3 t_{3}}{s_{4}}
\end{aligned}
$$




$$
\begin{aligned}
& +\frac{3 t_{3}}{s_{6}}-\frac{s_{1}}{s_{4}}-\frac{s_{3}}{s_{6}}-\frac{3 s_{4}}{s_{6}}+\frac{s_{6}^{2}}{s_{4} t_{3}}+\frac{s_{1}}{t_{3}}+\frac{s_{3}}{t_{3}}+\frac{s_{1} s_{4}}{s_{3} t_{3}}-\frac{s_{4} s_{5}}{s_{1} t_{3}}+\frac{s_{3} s_{6}}{s_{1} t_{3}}-\frac{s_{5} s_{6}}{s_{3} t_{3}} \\
& +\frac{s_{1} s_{6}}{s_{4} t_{3}}+\frac{s_{4} t_{1}}{s_{1} t_{3}}+\frac{s_{6} t_{1}}{s_{3} t_{3}}+\frac{s_{4} t_{2}}{s_{1} t_{3}}+\frac{s_{6} t_{2}}{s_{3} t_{3}}+\frac{s_{4}^{2}}{s_{6} t_{3}}+\frac{s_{3} s_{4}}{s_{6} t_{3}}+\frac{s_{1}}{s_{2}}+\frac{s_{3}}{s_{2}}+\frac{s_{5}}{s_{2}}+\frac{s_{6} t_{1}}{s_{4} s_{2}} \\
& \left.+\frac{s_{4} t_{2}}{s_{6} s_{2}}-\frac{t_{1} t_{3}}{s_{4} s_{2}}-\frac{t_{2} t_{3}}{s_{6} s_{2}}\right\}+\cdots .
\end{aligned}
$$

\section{References}

[1] J.D. Lykken, Weak scale superstrings, Phys. Rev. D 54 (1996) 3693, hep-th/9603133;

I. Antoniadis, N. Arkani-Hamed, S. Dimopoulos, G.R. Dvali, New dimensions at a millimeter to a Fermi and superstrings at a TeV, Phys. Lett. B 436 (1998) 257, hep-ph/9804398.

[2] I. Antoniadis, A possible new dimension at a few TeV, Phys. Lett. B 246 (1990) 377.

[3] D. Oprisa, S. Stieberger, Six gluon open superstring disk amplitude, multiple hypergeometric series and EulerZagier sums, hep-th/0509042.

[4] S. Stieberger, T.R. Taylor, Amplitude for $N$-gluon superstring scattering, Phys. Rev. Lett. 97 (2006) 211601, hep-th/ 0607184.

[5] S. Stieberger, T.R. Taylor, Multi-gluon scattering in open superstring theory, Phys. Rev. D 74 (2006) 126007, hep-th/ 0609175 .

[6] S. Stieberger, T.R. Taylor, Supersymmetry relations and MHV amplitudes in superstring theory, Nucl. Phys. B 793 (2008) 83, arXiv: 0708.0574 [hep-th].

[7] S. Cullen, M. Perelstein, M.E. Peskin, TeV strings and collider probes of large extra dimensions, Phys. Rev. D 62 (2000) 055012, hep-ph/0001166.

[8] E.H. Simmons, Dimension-six gluon operators as probes of new physics, Phys. Lett. B 226 (1989) 132;

E.H. Simmons, Higher dimension gluon operators and hadronic scattering, Phys. Lett. B 246 (1990) 471;

P.L. Cho, E.H. Simmons, Searching for G3 in $t$ anti- $t$ production, Phys. Rev. D 51 (1995) 2360;

H.K. Dreiner, A. Duff, D. Zeppenfeld, How well do we know the three gluon vertex? Phys. Lett. B 282 (1992) 441; L.J. Dixon, Y. Shadmi, Testing gluon selfinteractions in three jet events at hadron colliders, Nucl. Phys. B 423 (1994) 3, hep-ph/9312363;

L.J. Dixon, Y. Shadmi, Nucl. Phys. B 452 (1995) 724, Erratum.

[9] S.J. Parke, T.R. Taylor, An amplitude for $n$ gluon scattering, Phys. Rev. Lett. 56 (1986) 2459.

[10] M.L. Mangano, S.J. Parke, Multiparton amplitudes in gauge theories, Phys. Rep. 200 (1991) 301, hep-th/0509223.

[11] L.J. Dixon, Calculating scattering amplitudes efficiently, hep-ph/9601359.

[12] M.T. Grisaru, H.N. Pendleton, P. van Nieuwenhuizen, Supergravity and the S-matrix, Phys. Rev. D 15 (1977) 996; M.T. Grisaru, H.N. Pendleton, Some properties of scattering amplitudes in supersymmetric theories, Nucl. Phys. B 124 (1977) 81.

[13] S.J. Parke, T.R. Taylor, Perturbative QCD utilizing extended supersymmetry, Phys. Lett. B 157 (1985) 81;

S.J. Parke, T.R. Taylor, Phys. Lett. B 174 (1986) 465, Erratum.

[14] S.J. Parke, T.R. Taylor, Gluonic two goes to four, Nucl. Phys. B 269 (1986) 410.

[15] J. Polchinski, String Theory, Cambridge Univ. Press, 1998, Sections 6 and 12.

[16] T. Banks, L.J. Dixon, Constraints on string vacua with space-time supersymmetry, Nucl. Phys. B 307 (1988) 93; S. Ferrara, D. Lüst, S. Theisen, World sheet versus spectrum symmetries in heterotic and type II superstrings, Nucl. Phys. B 325 (1989) 501.

[17] D. Friedan, E.J. Martinec, S.H. Shenker, Conformal invariance, supersymmetry and string theory, Nucl. Phys. B 271 (1986) 93;

J. Cohn, D. Friedan, Z.a. Qiu, S.H. Shenker, Covariant quantization of supersymmetric string theories: The spinor field of the Ramond-Neveu-Schwarz model, Nucl. Phys. B 278 (1986) 577.

[18] V.A. Kostelecky, O. Lechtenfeld, W. Lerche, S. Samuel, S. Watamura, Conformal techniques, bosonization and tree level string amplitudes, Nucl. Phys. B 288 (1987) 173.

[19] M.L. Mangano, S.J. Parke, Z. Xu, Duality and multi-gluon scattering, Nucl. Phys. B 298 (1988) 653.

[20] D.A. Kosower, Next-to-maximal helicity violating amplitudes in gauge theory, Phys. Rev. D 71 (2005) 045007 , hep-th/0406175.

[21] R. Britto, F. Cachazo, B. Feng, New recursion relations for tree amplitudes of gluons, Nucl. Phys. B 715 (2005) 499, hep-th/0412308. 
[22] R. Britto, B. Feng, R. Roiban, M. Spradlin, A. Volovich, All split helicity tree-level gluon amplitudes, Phys. Rev. D 71 (2005) 105017, hep-th/0503198.

[23] M. Luo, C. Wen, Recursion relations for tree amplitudes in super gauge theories, JHEP 0503 (2005) 004, hep-th/ 0501121.

[24] S.J. Bidder, D.C. Dunbar, W.B. Perkins, Supersymmetric ward identities and NMHV amplitudes involving gluinos, JHEP 0508 (2005) 055, hep-th/0505249.

[25] F.A. Berends, W.T. Giele, Recursive calculations for processes with $n$ gluons, Nucl. Phys. B 306 (1988) 759.

[26] F. Cachazo, P. Svrcek, E. Witten, MHV vertices and tree amplitudes in gauge theory, JHEP 0409 (2004) 006, hep-th/ 0403047.

[27] R. Britto, F. Cachazo, B. Feng, E. Witten, Direct proof of tree-level recursion relation in Yang-Mills theory, Phys. Rev. Lett. 94 (2005) 181602, hep-th/0501052.

[28] S. Stieberger, T.R. Taylor, Non-Abelian Born-Infeld action and type I-heterotic duality. I: Heterotic $F^{6}$ terms at two loops, Nucl. Phys. B 647 (2002) 49, hep-th/0207026;

S. Stieberger, T.R. Taylor, Non-Abelian Born-Infeld action and type I-heterotic duality. II: Nonrenormalization theorems, Nucl. Phys. B 648 (2003) 3, hep-th/0209064. 